\title{
Isotopic and spin-nuclear effects in solid hydrogens
}

\author{
(Review Article)
}

\author{
Yuri A. Freiman \\ B. Verkin Institute for Low Temperature Physics and Engineering of the National Academy of Sciences of Ukraine \\ 47 Nauky Ave., Kharkiv 61103, Ukraine \\ yuri.afreiman@gmail.com
}

\section{Yanier Crespo}

International Center for Theoretical Physics (ICTP), IT-34014 Trieste, Italy

E-mail: yanier@hotmail.com

Received April 2, 2017, published online October 25, 2017

The multiple isotopic family of hydrogens $\left(\mathrm{H}_{2}, \mathrm{HD}, \mathrm{D}_{2}, \mathrm{HT}, \mathrm{DT}, \mathrm{T}_{2}\right)$ due to large differences in the de Boer quantum parameter and inertia moments displays a diversity of pronounced quantum isotopic solid-state effects. The homonuclear members of this family $\left(\mathrm{H}_{2}, \mathrm{D}_{2}, \mathrm{~T}_{2}\right)$ due to the permutation symmetry are subjects of the constraints of quantum mechanics which link the possible rotational states of these molecules to their total nuclear spin giving rise to the existence of two spin-nuclear modifications, ortho- and parahydrogens, possessing substantially different properties. Consequently, hydrogen solids present an unique opportunity for studying both isotope and spin-nuclear effects. The rotational spectra of heteronuclear hydrogens (HD, HT, DT) are free from limitations imposed by the permutation symmetry. As a result, the ground state of these species in solid state is virtually degenerate. The most dramatic consequence of this fact is an effect similar to the Pomeranchuk effect in ${ }^{3} \mathrm{He}$ which in the case of the solid heteronuclear hydrogens manifests itself as the reentrant broken symmetry phase transitions. In this review article we discuss thermodynamic and kinetic effects pertaining to different isotopic and spin-nuclear species, as well as problems that still remain to be solved.

\section{PACS: 67.80.ff Molecular hydrogen and isotopes.}

Keywords: isotopic family of hydrogens, homo- and heteronuclear species, ortho- and parahydrogens, isotope and spin-nuclear effects, ortho- and para conversion, Pomeranchuk effect, reentrant broken symmetry phase transition.

\section{Contents}

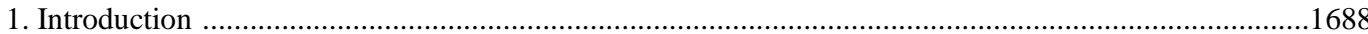

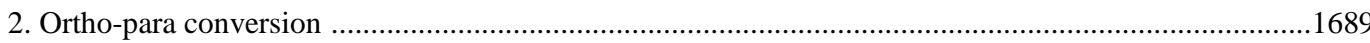

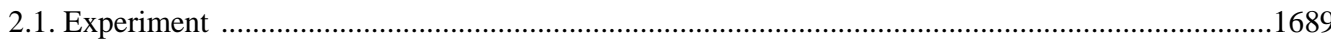

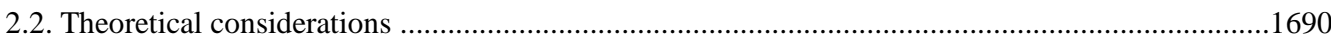

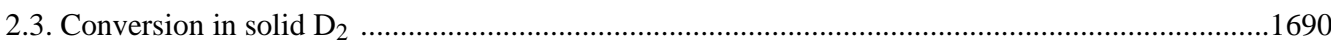

2.4. Validity of the concept of ortho-para states at high pressure .......................................................1691

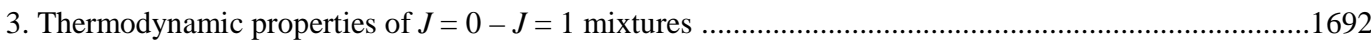

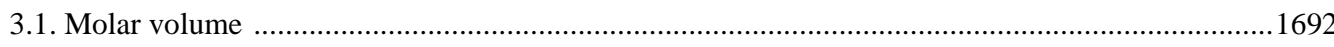

3.2. HCP lattice distortion in solid $\mathrm{H}_{2}$ as a function of $o-p$ composition .............................................1692

3.3. Experimental determination of the crystal field splitting of isolated $J=1$ impurities in solid

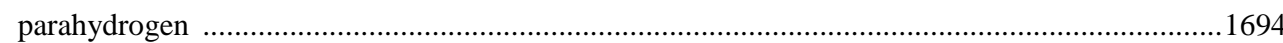

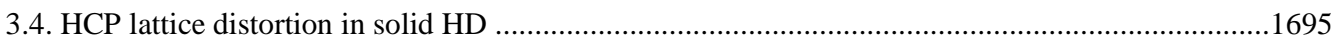

4. Broken symmetry phase transition in solid HD and $o-p$ mixtures ......................................................1695

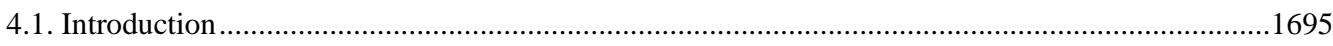

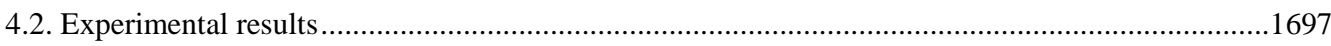


4.3. Theoretical studies

4.3.1. Quantum orientational melting in all- $J$ system ... 1698

4.3.2. The reentrant behavior from numerical simulations

4.4. Reentrant phase transitions in ortho-para mixtures

\section{Introduction}

The large isotopic family of hydrogens $\left(\mathrm{H}_{2}, \mathrm{HD}, \mathrm{D}_{2}\right.$, $\mathrm{HT}, \mathrm{DT}, \mathrm{T}_{2}$ ) presents an unique possibility for studying the diversity of quantum isotopic effects [1-3]. The differences in properties cannot be, as a rule, related solely to the de Boer quantum parameter, since symmetry-related nuclear spin effects turn out to be far more essential.

The requirements of quantum mechanics on a homonuclear hydrogen species $\left(\mathrm{H}_{2}, \mathrm{D}_{2}, \mathrm{~T}_{2}\right)$ rigidly link the rotational momentum $J$ and the total nuclear spin $I_{\text {mol }}$ of the molecule. According to the principle of the indistinguishability of identical particles, the molecular wave function of any homonuclear diatomic molecule must be symmetric or antisymmetric under nuclear exchange. The nucleus of hydrogen atom is fermion with nuclear spin $I_{N}=1 / 2$ and the nucleus of deuterium atom is boson with nuclear spin $I_{N}=1$. So the total molecular wave function, which is a product of vibrational, spin, and rotational wave functions, $\psi_{\text {mol }}=\psi_{\text {vib }} \psi_{\text {spin }} \psi_{\text {rot }}$, must be antisymmetric for the hydrogen molecule and symmetric for the deuterium molecule. The vibrational part, $\psi_{\text {vib }}$, is always symmetric. The spin part, $\psi_{\text {spin }}$, for the hydrogen molecule is antisymmetric for the singlet state $I_{\text {mol }}=0$, and symmetric for the triplet state $I_{\text {mol }}=1$. Hence the rotational part $\psi_{\text {rot }}$ is symmetric (rotational quantum number $J=0,2,4, \ldots$ ) for the singlet state and antisymmetric $(J=1,3, \ldots)$ for the triplet state. For the deuterium molecule the spin part is symmetric for $I_{\mathrm{mol}}=2 ; 0$ and antisymmetric for $I_{\mathrm{mol}}=1$. The rotational part $\psi_{\text {rot }}$ respectively is symmetric ( $J=0$ or even) for the symmetric spin state $I_{\mathrm{mol}}=2 ; 0$ state and antisymmetric ( $J_{\text {odd }}$ ) for the $I_{\text {mol }}=1$ spin state. The states with the parity of the largest possible $I_{\text {mol }}$ value are called ortho while the states with the other parity are para. Transitions between states of different parity ( $J$ or $\left.I_{\mathrm{mol}}\right)$, an ultimately quantum process, called ortho-para conversion, are strictly forbidden in a single molecule and is a low-probability process in a condensed state. This enables treating the ortho- and paraspecies as rather stable different substances (orthohydrogen, $o-\mathrm{H}_{2}$, and parahydrogen, $p-\mathrm{H}_{2}$, and their isotopic counterparts $p-\mathrm{D}_{2}$ and $o-\mathrm{D}_{2}$ ) with their own properties.

The equilibrium ortho-para composition in the mixtures can be calculated by using the rotational energy of a diatomic rotator $E_{\text {rot }}=B_{\text {rot }} J(J+1)$, where $B_{\text {rot }}$ is the rotational constant (85.25 K for $\mathrm{H}_{2}$ and $42.97 \mathrm{~K}$ for $\mathrm{D}_{2}$ [3]), and assuming a Boltzmann distribution. At high temperature the equilibrium ratio of ortho- to para species is determined by their statistical weights and is therefore 3:1 for hydrogen and 2:1 for deuterium. Such mixtures are called normal $\left(n-\mathrm{H}_{2}\right.$ and $\left.n-\mathrm{D}_{2}\right)$. The thermodynamic equilibrium ortho-para concentration for $\mathrm{H}_{2}$ and $\mathrm{D}_{2}$ is shown in Fig. 1 . Thus the constraints of quantum mechanics link the possible rotational states of the hydrogen molecule to its total nuclear spins. This symmetry-related link imparts large differences in the solid-state properties of ortho- and para modifications and may be considered as one of the most striking macroscopic manifestations of the microscopic laws of quantum mechanics. Due to this unique relation between the rotational and spin quantum numbers of homonuclear molecules the transitions between $J$-even and $J$-odd rotational states with $\Delta J= \pm 1, \pm 3, \ldots$ require the simultaneous change $\Delta I_{\mathrm{mol}}= \pm 1$ of the total nuclear spin of the molecule. Resulting transformation of the two species into each other is called ortho-para conversion. In isolated molecules the probability of such transitions is negligible and they may be considered as rigorously forbidden. The conversion in solid hydrogen and deuterium will be considered in Sec. 2 .

In contrast to $\mathrm{H}_{2}$ and $\mathrm{D}_{2}$, the nuclei in the heteronuclear HD molecule are distinguishable, and no symmetry requirements exist on the nuclear wave functions of HD. As a result, the heteronuclear molecules do not have orthopara species, and for both possible total nuclear spin $I_{\text {mol }}=1 / 2,3 / 2$ all angular momentum states $J=0,1,2, \ldots$ and transition between them are allowed.

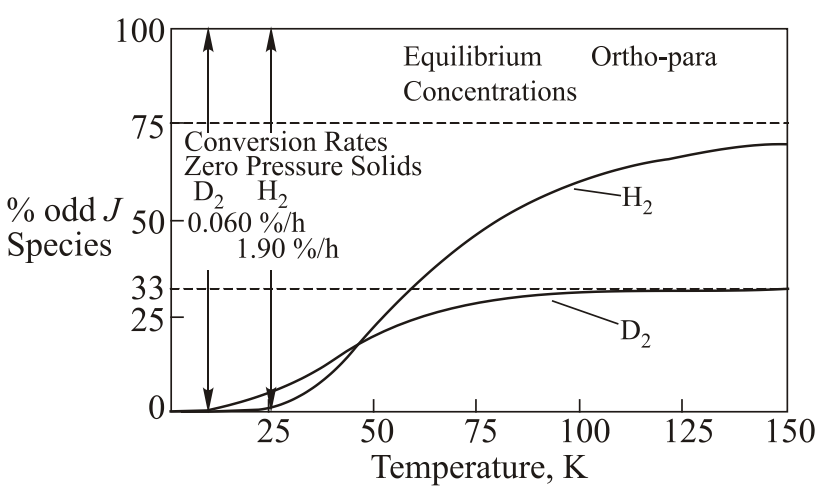

Fig. 1. Equilibrium ortho-para concentration of noninteracting hydrogen and deuterium as a function of temperature from Ref. 1. 


\section{Ortho-para conversion}

\subsection{Experiment}

While ortho-para conversion is strictly forbidden for isolated molecules, conversion does occur in solid. The ortho-para conversion in solid $\mathrm{H}_{2}$ was first studied experimentally as far back as in the beginning of the 1930s by Cremer and Polany [4,5] soon after the discovery of the two hydrogen modifications. At ambient pressure ortho-para conversion in the solid is very slow, taking weeks for a sample to equilibrate [1,2]. It was found that the conversion reaction is autocatalytic, that is, the rate of the reaction is determined by the average number of nearest $o-\mathrm{H}_{2}$ neighbors $M$ surrounding each $o-\mathrm{H}_{2}$ molecule: $d x / d t=-M x$, where $x$ is the $o-\mathrm{H}_{2}$ concentration. As a result, for the normal equilibrium distribution the rate equation describing the time dependence of the ortho concentration is

$$
d x / d t=-k x^{2}
$$

where $k$ is the macroscopic conversion constant. The analytic solution of this equation is $x^{-1}-x_{0}^{-1}=k t$, which can be used for comparison with experimental results.

There have been numerous experimental studies of the ortho-para conversion rate at pressures below 1 GPa by using various methods [6-12]. Ahlers [7] determined the conversion rate by measurement of the heat conduction of a gaseous sample before and after solidification. Pedroni et al. [8] and Schmidt [9] used nuclear magnetic resonance (NMR): the ortho molecules with $I_{\text {mol }}=I_{1}=1$ contribute to the nuclear magnetization and thus to the NMR signal, while the para molecules with $I_{\text {mol }}=I_{0}=0$ do not. Silvera's group [6,11,12] used Raman measurements. The ortho concentration was determined by comparing the relative intensities $I_{0}$ and $I_{1}$ of the respective Raman-active rotational transitions $J=0 \rightarrow 2$ and $J=1 \rightarrow 3$. At low temperatures $k_{B} T<<6 B$, the respective equation takes the form [13]

$$
x=\frac{5}{3}\left(I_{0} / I_{1}+\frac{5}{3}\right)^{-1} .
$$

The experimental data on the conversion rate as a function of density at small compressions up to $\rho / \rho_{0} \approx 1.7$ from Refs. 6-12 are shown in Fig. 2. Here $\rho_{0}$ is zeropressure density and $\rho$ is the density at pressure $P$. The experimental results are seen to be mutually consistent over the whole pressure range.

The most extensive measurements at zero pressure were made by Schmidt by NMR at three temperatures 12, 4.2, and $1.57 \mathrm{~K}$ in long-term experiment up to $900 \mathrm{~h}$. He gets a conversion rate of $k=(1.90 \pm 0.03) \% / h$ which is in agreement with most of the previous experiments. Measurements at small pressures showed that the conversion rate monotonously increases with pressure. Driessen et al. [6] found a maximum conversion rate at $0.5 \mathrm{GPa}$ followed by a drop up to 0.7 GPa (Fig. 2).

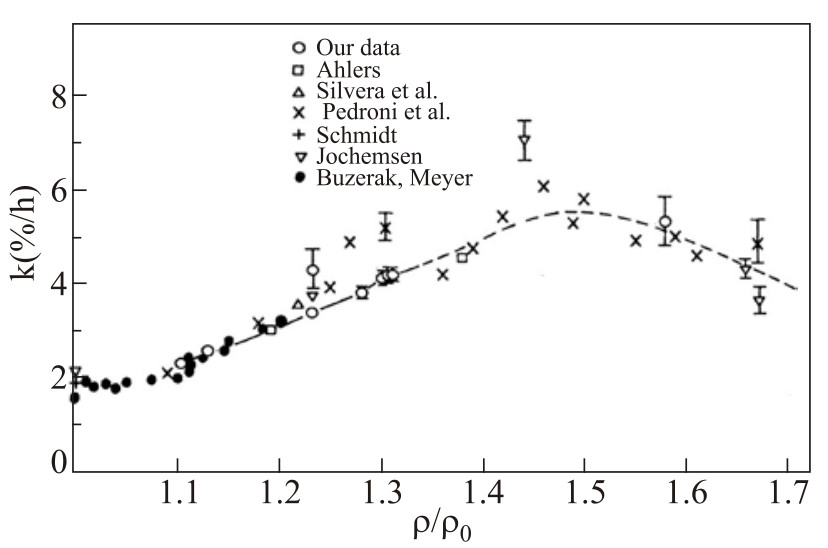

Fig. 2. The conversion rate in solid $\mathrm{H}_{2}$ as a function of the reduced density at small compressions (after Ref. 6).

Phase diagram studies at high pressures have inspired a renewed interest in the ortho-para conversion in solid $\mathrm{H}_{2}$. Hemley et al. [13] using Raman method raised the pressure limit in the conversion measurements up to $58 \mathrm{GPa}$. Several characteristic low-temperature $(10 \mathrm{~K})$ spectra at $21.6 \mathrm{GPa}$ are given in Fig. 3. They show the decrease in the integrated intensity of the $S_{0}(1)$ peak with respect to the $S_{0}(0)$ peak with time (here the roton bands $S_{0}(0)$ and $S_{0}(1)$ involve transitions $J=0 \rightarrow J=2, J=1 \rightarrow J=3$, respectively). A rather intricate non-monotonous pressure dependence of the conversion rate emerged from these studies (Fig. 4).

Following maximum at about $0.5 \mathrm{GPa}$ the conversion slows to a minimum at about 3 GPa nearly a factor of two below the ambient pressure rate constant. At higher pressures the conversion rate increases rapidly to about $260 \% / \mathrm{h}$ at $58 \mathrm{GPa}$. The results are compared with NMR measurements to $12.8 \mathrm{GPa}[15]$ ( $\rho / \rho_{0} \approx 3.7$ ). On the whole, there is an excellent agreement between results obtained by the

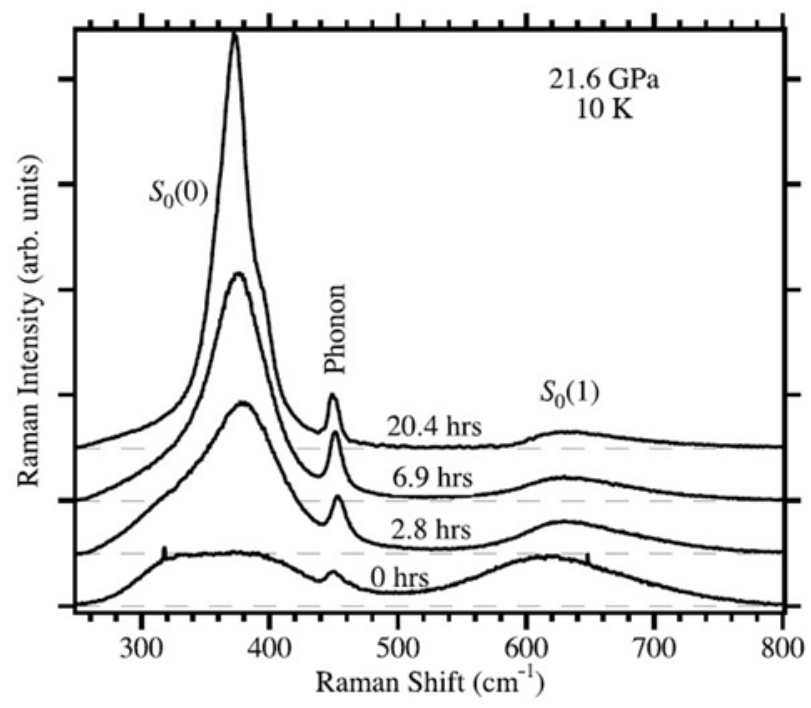

Fig. 3. Changes in the Raman spectra of solid $\mathrm{H}_{2}$ at $21.6 \mathrm{GPa}$ and $10 \mathrm{~K}$ with time elapsed from cool-down completion (after Eggert et al. [14]). 


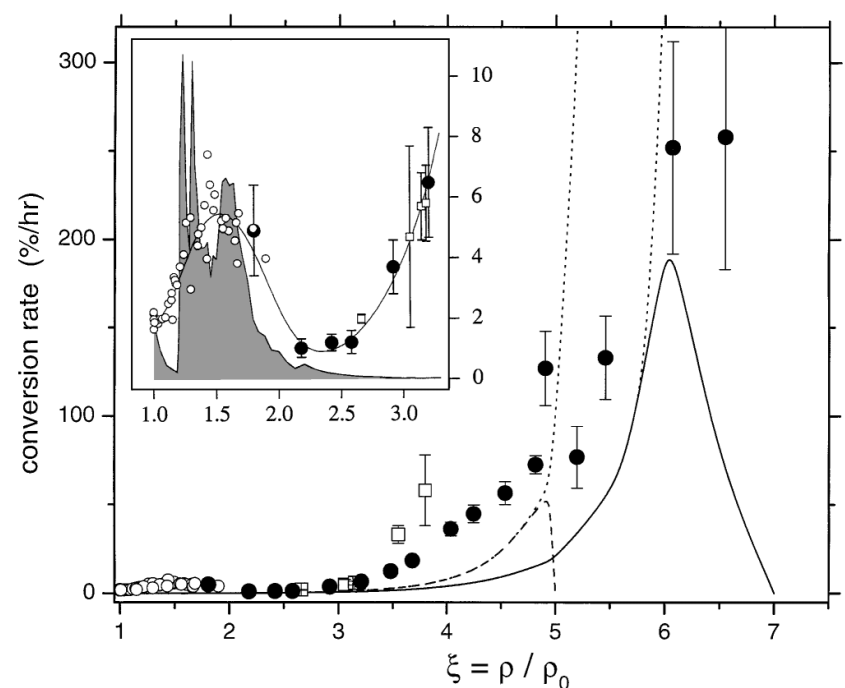

Fig. 4. The conversion rate vs. reduced density (the crystal density ratio reduced to the $P=0$ value) at high compression. Solid circles are from Refs. 13, 14, empty squares are from Ref. 15. The solid curve is the theoretical prediction for hcp $n-\mathrm{H}_{2}$ from Ref. 16. The open circles are earlier data (see Fig. 2). Inset: Low density region (see Ref. 14). After Eggert et al. [14].

two methods with possible discrepancies arising from temperature differences in the two studies.

\subsection{Theoretical considerations}

A theory of the ortho-para conversion in solid hydrogen was first developed by Motizuki and Nagamiya [17]. They showed that the conversion rate is controlled by the three mechanisms: (1) the magnetic dipole-dipole interaction of the nuclear spins of ortho molcules; (2) magnetic-dipole interaction between the nuclear spins and the rotational magnetic moments; (3) magnetic interaction with paramagnetic impurities.

The conversion transition ratio $W$ can be calculated from the golden rule:

$$
W=\frac{2 \pi}{\hbar} \sum_{\text {if }} P_{i}\left|\left\langle f\left|\mathcal{H}_{\text {int }}\right| i\right\rangle\right|^{2} \delta\left(E_{i}-E_{f}\right),
$$

where $P_{i}$ is the probability that the system is initially in one of the states $i$, and $f$ is the final state; $E_{i}$ and $E_{f}$ are the initial and final energies of the system. $\mathcal{H}_{\text {int }}$ is the interaction Hamiltoman and has three additive parts which can change the nuclear spin states: $\mathcal{H}_{s s}$, the nuclear spinspin interaction; $\mathcal{H}_{r s}$, the rotation-spin interaction; and $\mathcal{H}_{q q}$ , the electric quadrupole interaction, (explicit equations can be found in Refs. 3, 18). $\mathcal{H}_{s s}$ is the dominant term with $\mathcal{H}_{\text {rs }}$ yielding a $2-3 \%$ correction to $\mathcal{H}_{s s}$. The delta-function in Eq. (3) requires conservation of energy. The rotational energy change in a conversion is $B_{\text {rot }} J(J+1)$. According to the theoretical description of ortho-para conversion in solid hydrogen as developed by Motizuki and Nagamiya [17], an ortho-para transition requires a large magnetic field gradient acting on a molecule to flip a proton spin. This field gradient is supplied by the rotational magnetic moment of neighboring ortho molecules. Thus two ortho-neighbors are needed for one of them to convert. As a result, if the ortho distribution is random, the rate equation Eq. (1) describes the time dependence of the ortho concentration.

The rotational energy difference between a $J=1$ ortho molecule and a $J=0$ para molecule, $\Delta E_{0 \leftarrow 1}=2 B_{\text {rot }}=$ $=171 \mathrm{~K}$, released by ortho-para conversion must be absorbed by the lattice. The Debye temperature $\Theta_{D}$ gives an estimate for the limiting phonon energy. Since at ambient pressure $\Theta_{D}=118.5 \mathrm{~K}$ [3] the energy conservation requires the simultaneous creation at least two phonons. The two-phonon theory of Motizuki and Nagamiya predicts a drop in the conversion rate with increasing pressure over about 1 kbar where the two-phonon conversion rate is negligible. Thus, neither the two-phonon theory nor the onephonon theory in the harmonic approximation can explain experimental results $[7,8,10]$ which showed the conversion rate to be an increasing function of density. Therefore Berlinsky [19] suggested that the effect of the large anharmonisity in solid hydrogen is to broaden the high-energy features of the phonon spectrum into a high-energy tail which extends far above $k_{B} \Theta_{D}$. On the basis of this conjecture Berlinsky [19] developed a detailed theory of onephonon processes and found that the one-phonon conversion rate should initially increase with pressure before falling above about $0.5 \mathrm{GPa}$ because of density of states factors and the rapid increase in phonon energy.

On the base of the conversion rate data $[14,15]$ a new conversion mechanism was proposed by Strzhemechny and Hemley in Ref. 16 and by Strzhemechny et al. in Ref. 20 that differs from that employed to explain low-pressure data. The steep increase in conversion rates at high pressures was explained by a conversion channel that involves an intermediate state in which new excitations are created due to the electric quadrupole-quadrupole (EQQ) coupling between rotational momenta. The enhancement was explained by a gap closing that arises when the EQQ interaction becomes sufficient to substantially diminish the conversion energy released because of the lowering of the groundstate level of the converted ortho molecule. It was shown that this concentration-sensitive channel comes into play at compressions $\xi=\rho / \rho_{0}$ between 3 and 4 and ceases to operate at higher values $(\xi \approx 6-7)$, depending on the ortho concentration. Thus, this theory predicts a significant reduction in the conversion rate at about $80 \mathrm{GPa}$; The extension of the measurements to pressures over $50 \mathrm{GPa}$ would provide a critical test of the conversion mechanisms proposed in Refs. 16, 20.

\subsection{Conversion in solid $\mathrm{D}_{2}$}

Theory of conversion in solid $\mathrm{D}_{2}$ was developed first by Motizuki [21]. The conversion in $\mathrm{D}_{2}$ differs from that in $\mathrm{H}_{2}$ in a number of essential aspects. First of all, the deuterium nucleus, deuteron, possesses a nonzero quadrupole 
moment. The interaction of this quadrupole moment with electric field gradients due to neighboring molecules provides another mechanism for conversion in addition to dipolar interactions. Also, the deuteron has $I_{D}=1$, so the final state of the $J=0 \mathrm{D}_{2}$ molecule may be any of the six nuclear states corresponding to $I=0$ or 2 . If the final nuclear state is $I=2$, then this molecule will cause magnetic field gradients at neighboring molecules, and thus it may still contribute to the conversion process. This is in contrast to the case of solid $\mathrm{H}_{2}$ where the para molecules have $J=0, I_{\text {mol }}=0$ and thus do not contribute to further conversion.

One more significant difference between the conversion in $\mathrm{D}_{2}$ and $\mathrm{H}_{2}$ lies in the fact that the rotational constant of $\mathrm{D}_{2}$, $B_{\text {rot }}^{\mathrm{D}_{2}}=42.97 \mathrm{~K}$ [3], about a half of that for $\mathrm{H}_{2}(85.25 \mathrm{~K})$ with the respective change of the rotational energy in going from the $J=1$ to the $J=0$ state, while the Debye temperature $\left(\Theta_{D}^{D_{2}}\right)=114.1 \mathrm{~K}$ [3] is nearly the same $\left(\Theta_{D}^{\mathrm{H}_{2}}\right)=$ $=118.5 \mathrm{~K}$ [3]. Thus the one-phonon process is allowed for conversion in solid $\mathrm{D}_{2}$.

The presence of the conversion mechanism that involves $J=0$ molecules with $I=2$ changes the rate equation describing the time dependence of the concentration of $J=1$ molecules:

$$
d x / d t=-k x^{2}-k^{\prime} x(1-x) .
$$

The general solution of this equation with the initial condition $x(t=0)=x_{0}$ has the form

$$
x^{-1}=x_{0}^{-1}-\left(x_{0}^{-1}-1+k / k^{\prime}\right)\left[1-\exp \left(k^{\prime} t\right)\right]
$$

A characteristic distinction of the conversion in deuterium is that at low $J=1$ fraction the conversion rate is linear in the concentration $x$ of the $J=1$ molecules. Experimental results obtained by different methods (see Table 1) gave rather close results:

Table 1. The conversion constants in solid deuterium at $P=0$

\begin{tabular}{l|c|c}
\hline \hline \multicolumn{1}{c|}{ Author, Ref., Method } & $k\left(10^{-4} \mathrm{~h}^{-1}\right)$ & $k^{\prime}\left(10^{-4} \mathrm{~h}^{-1}\right)$ \\
\hline \hline $\begin{array}{l}\text { Hardy and Berlinsky [22] (NMR) } \\
\text { Milenko and Sibileva [23] }\end{array}$ & 5.6 & $k=k^{\prime}$ \\
$\begin{array}{l}\text { Berkhout, Minneboo, and Silvera } \\
\text { [24] (Raman) }\end{array}$ & $6.3 \pm 0.1$ & $k=k^{\prime}$ \\
$\begin{array}{l}\text { Calkins, Banke, Li, and Meyer [25] } \\
\text { (NMR) }\end{array}$ & $5.5 \pm 0.5$ & $k=k^{\prime}$ \\
$\begin{array}{l}\text { Bagatskii, Krivchikov, Manzhelii } \\
\text { et al. [26] }\end{array}$ & $6.4 \pm 0.2$ & \\
$\begin{array}{l}\text { Strzhemechny and Tokar [27] } \\
\text { (Theory) }\end{array}$ & 6.1 & 14.2 \\
\hline \hline
\end{tabular}

Comparison with the conversion rate in $\mathrm{H}_{2}\left(1.9 \cdot 10^{-2} \mathrm{~h}^{-1}\right)$ shows that in $\mathrm{D}_{2}$ it proceeds about 30 times slower. The efficiency of various conversion channels in solid deuterium at high pressures have been considered by Strzhemechny and Hemley [28]. They found that the standard phonon- assisted channels are inefficient at high pressures in $\mathrm{D}_{2}$ as they are in $\mathrm{H}_{2}$, and the idea of the intermediate state with subsequent participation of the EQQ interaction remains productive for $\mathrm{D}_{2}$.

\subsection{Validity of the concept of ortho-para states at high pressure}

At small pressures when the wave function of the crystal may be represented as a product of molecular wave functions there are no any doubts that the notion of orthopara states is a well justified quantum-mechanical concept. To what extent this concept remains valid at high pressures when the rotational quantum number $J$ is no longer a good quantum number? How does an essential increase in the conversion rate with pressure affect the ortho-para concept? These important questions were analyzed in detail by Silvera [29] and Silvera and Pravica [30]. Their main conclusion is that the only important quantum number for the $o-p$ states is parity under exchange and that the nuclear spin states preserve up to pressures when the dissociative Wigner-Huntington transition occurs.

In solid molecular hydrogen or deuterium the manybody wave function must be symmetrized with respect to exchange of nucleons. Nucleon exchange can take place within a molecule or between molecules. If exchange of nucleons between neighboring molecules took place, then the structure of the wave functions under nucleon permutation would be very complicated and the ortho-para concept associated with isolated molecules would not be applicable.

The total molecular wave function can be written as a superposition of pure ortho and pure para states:

$$
\Psi=\Psi_{\text {ortho }}+\alpha \Psi_{\text {para }} .
$$

For the validity of the concept of ortho-para species, the mixing parameter $\alpha$ should be very small.

The perturbation which causes mixing is the same that causes conversion. Taking into consideration the mechanism of Motizuki and Nagamiya Silvera obtained the following density dependence of the resultant mixing parameter [29]:

$$
|\alpha|=8 \cdot 10^{-10} c_{1}\left(\rho / \rho_{0}\right)^{4 / 3} .
$$

where $c_{1}$ is the ortho concentration. From Eq. (7) we see that at zero pressure the mixing parameter is exceptionally small. The highest compression $\xi=\rho / \rho_{0}$ reached in the equation of state experiments at $180 \mathrm{GPa}$ is 10.4 [31]. The mixing parameter increases by a factor 22.7 but remains very small of order $10^{-8}$, and the concept of ortho-para species remains valid to very high pressure.

There is an interesting question concerning a possible implication of ortho-para species for metallization [30]. At a finite temperature we have a mixture of ortho- and parastates which violates the translational invariance. This can possibly give rise to Anderson localization and sup- 
press an insulator-metal transition that would occur in a pure lattice [32]. It might be the case that even pure orthoor para lattices suffer from Anderson localization. Since the electron motions are very much faster than the nuclear motions, an electron moving through a pure lattice sees a snapshot of molecules with random orientations, although on the average (in the quantum mechanical sense), the molecules have well defined distributions. At this time it is not clear from theoretical considerations whether the electron feels a translationally invariant or a disordered lattice. It is possible that the failure to observe metallization in the phase III of hydrogen, above $150 \mathrm{GPa}$, is due to this type of disorder.

\section{Thermodynamic properties of $\boldsymbol{J}=\mathbf{0}-\boldsymbol{J}=\mathbf{1}$ mixtures}

\subsection{Molar volume}

In 1972 Silvera et al. [33] studying Raman-active $E_{2 g}$ phonon mode in hcp hydrogen and deuterium found unexpectedly large downward shift $(\sim 5 \%)$ in the measured frequency when going from pure $J=1$ to pure $J=0$ solid. This effect was explained by Silvera et al. [12] by the difference in molar volume of $J=1$ and $J=0$ species, the former having a smaller molar volume. The free energy has translational (lattice) contributions and orientational contributions related with isotropic and anisotropic interactions, respectively. When compressions are not too high, we may restrict ourselves to the contribution from EQQ (electric quadrupole-quadrupole) interactions. The quadrupole pressure difference between $J=1$ and $J=0 \mathrm{H}_{2}$ and $\mathrm{D}_{2}$ in the orientationally ordered $\mathrm{Pa} 3$ state is shown in Fig. 5 [11]. Hydrogen is a quantum solid and is therefore highly compressible. Due to the large compressibility, the weak anisotropic forces related with $J=1$ species have a non-negligible effect on the molar volume. The zero-

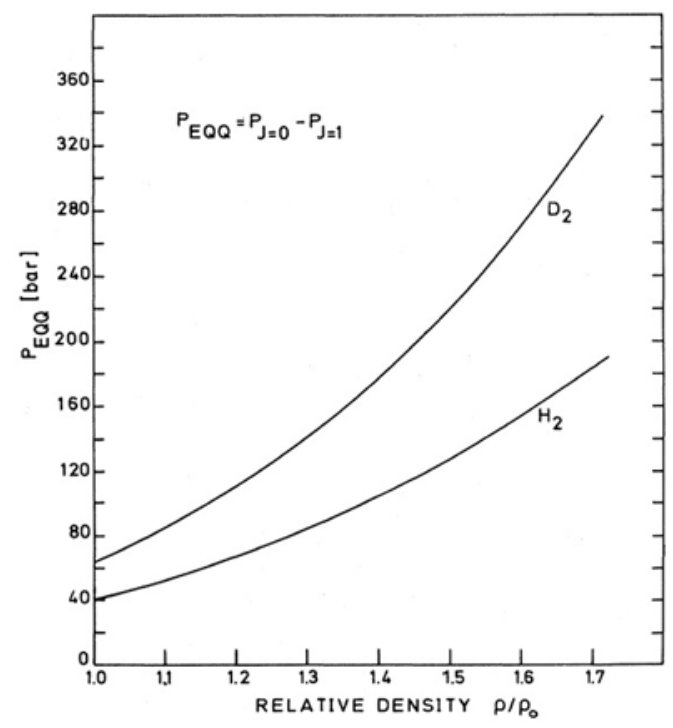

Fig. 5. The quadrupole pressure in $p-\mathrm{H}_{2}$ and $o-\mathrm{D}_{2}$ in $\mathrm{Pa} 3$ ordered state at $T=0$ (after Jochemsen et al. [11]). pressure molar volume $V$ of hydrogen and deuterium as a function of $J=1$ concentration is shown in Fig. 6. In the orientationally disordered state of hexagonal close packed (hcp) solids at $T=4.2 \mathrm{~K}$ we have the following dependencies [1]: $V(x)=23.16-0.091 x-0.217 x^{2} \mathrm{~cm}^{3} / \mathrm{mol}\left(\mathrm{H}_{2}\right)$, and $V(x)=19.95-0.16 x-0.04 x^{2} \mathrm{~cm}^{3} / \mathrm{mol}\left(\mathrm{D}_{2}\right)$.

\subsection{HCP lattice distortion in solid $\mathrm{H}_{2}$ as a function of o-p composition}

At zero pressure the molecules in $J$-even solid hydrogens $\left(p-\mathrm{H}_{2}, o-\mathrm{D}_{2}\right)$ are virtually spherical. Rigid spheres in the undistorted lattice crystallize into fcc (face center cubic) or hcp lattice. As compared with fcc, the hcp lattice has an additional degree of freedom associated with the $c$ / $a$ ratio. A lattice of closed packed hard spheres has $c / a=\sqrt{8 / 3} \approx 1.633$ (an ideal hcp structure). The quantity $\delta=c / a-\sqrt{8 / 3}$, the lattice distortion parameter, describes the deviation of the axial ratio from the ideal value. In the case of $\delta<0$, this distortion involves extension within close-packed planes, and contraction along the $c$-axis direction, and vice versa, for $\delta>0$ the lattice is expanded along the $c$ axis and contracted within close-packed planes.

Calculations with pair isotropic potentials have shown that the ideal hcp lattice does not correspond to minimal lattice energy [35-37]. Calculations with a many-body potential and DFT calculations performed for solid He showed [38] that the pressure dependencies of the lattice distortion parameter $\delta(P)$ for a many-body (two- plus three-body) and for pair intermolecular potentials are qualitatively different. The three-body forces flatten the lattice $(\delta<0)$ while the pair forces at large compressions tend to elongate it $(\delta>0)$. Thus, it was shown that the lattice distortion parameter is a thermodynamic characteristic which is very sensitive to the many-body component of the intermolecular potential and can therefore be used as a probe of the many-body forces [39].

The deviation of the axial ratio from the ideal value in the hcp solids can be attributed ultimately to a lowering of the band-structure energy through lattice distortion. In the molecular hcp solids we can explicitly ascribe this effect to the reduction in the ground-state energy due to lattice distortion. In the case of solid hydrogens the effect of lattice distortion both on the isotropic and rotational components of the zero-point energy is essential.

The orientational state of molecules in the distorted lattice is characterized by the orientational order parameter $\eta=\sqrt{4 \pi / 5}\left\langle Y_{20}\right\rangle$, where $\langle(\ldots)\rangle$ means thermodynamic averaging with the rotational Hamiltonian. The latticerotation coupling is described by the the Hamiltonian $\mathcal{H}_{\text {int }}=-\varepsilon_{2 c} \sqrt{4 \pi / 5} Y_{20}$, where $\varepsilon_{2 c}$ is the crystal-field parameter [2] which is linear with respect to $\delta$ :

$$
\varepsilon_{2 c}=\tilde{B} \delta ; \tilde{B}=-\sqrt{6}\left(B+\frac{1}{2} R \frac{d B}{d R}\right),
$$




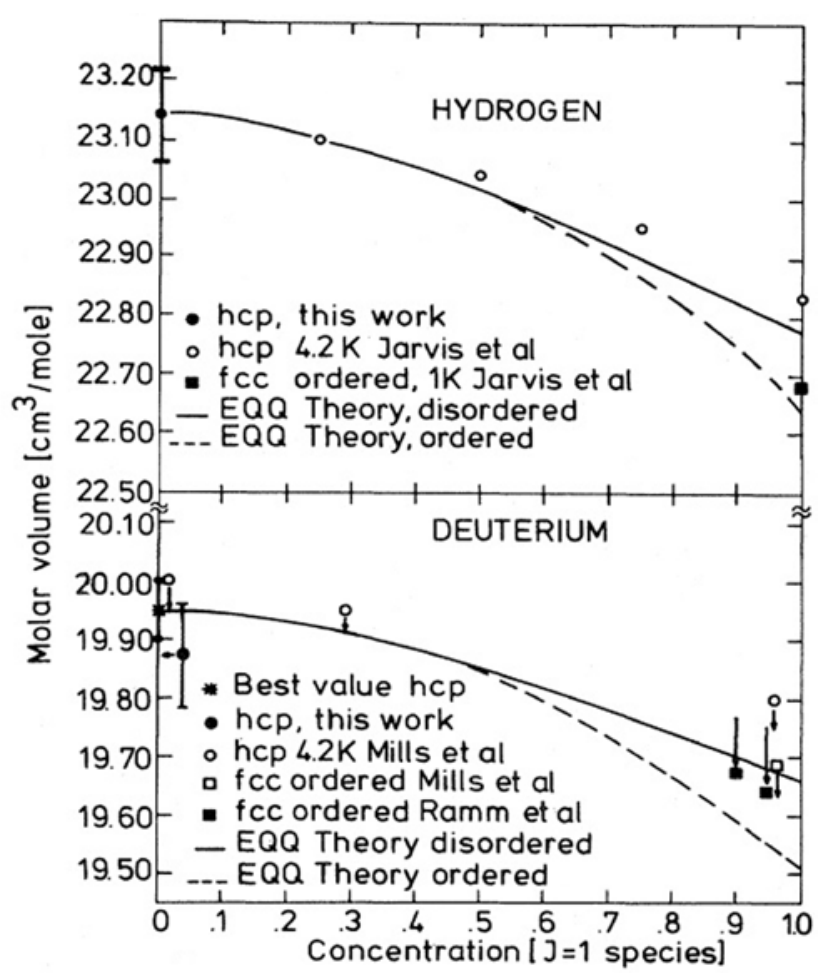

Fig. 6. The molar volume at zero pressure as a function of concentration of $J=1$ modification (after Driessen et al. [34]).

where $B(R)$ is the radial function of the single-molecular term in the anisotropic intermolecular potential [2]. Thus, the state of the lattice can be described by two coupled order parameters, $\eta(P, T)$ and $\delta(P, T)$, which can be found by the minimization of free energy with respect to these parameters. Figure 6 shows the volume dependence of the lattice distortion parameter $\delta$ and orientational order parameter $\eta$ in parahydrogen and orthodeuterium calculated using the many-body intermolecular potential from Ref. 40. The negative sign of $\delta$ means that the lattice is flattened compared with the ideal one.

For all hcp elemental solids except helium, hydrogen, and high-pressure $\mathrm{Ar}, \mathrm{Kr}$, and $\mathrm{Xe}$ the behavior of $\delta$ with pressure and temperature is well established from both theory and experiment. Typical values are of the order of $10^{-2}$. For solid helium $\delta$ is an order of magnitude smaller $[38,41]$. The first measurements of $c / a$ ratio in solid hydrogens were done by Keesom et al. [42] who found that at zero pressure hcp lattice of $p-\mathrm{H}_{2}$ is close to ideal. X-ray zero-pressure study by Krupskii et al. [43] confirmed this result $(c / a=1.633 \pm 0.001)$ and extended it for $o-\mathrm{D}_{2}$. In fact, the only structural study of $p-\mathrm{H}_{2}$ and $o-\mathrm{D}_{2}$ at elevated pressures up to $2.5 \mathrm{GPa}$ and low temperatures were made by Ishmaev et al. using the neutron diffraction method [44,45]. It was found that the ratio $c / a$ is practically constant and is slightly less than the ideal hcp value $(1.631 \pm 0.002)$.

In the absence of direct experimental data some qualitative conclusions on the lattice distortion parameter of $p-\mathrm{H}_{2}$ were obtained by Goncharov et al. [46] from low-frequency Raman spectra at low temperature. The authors

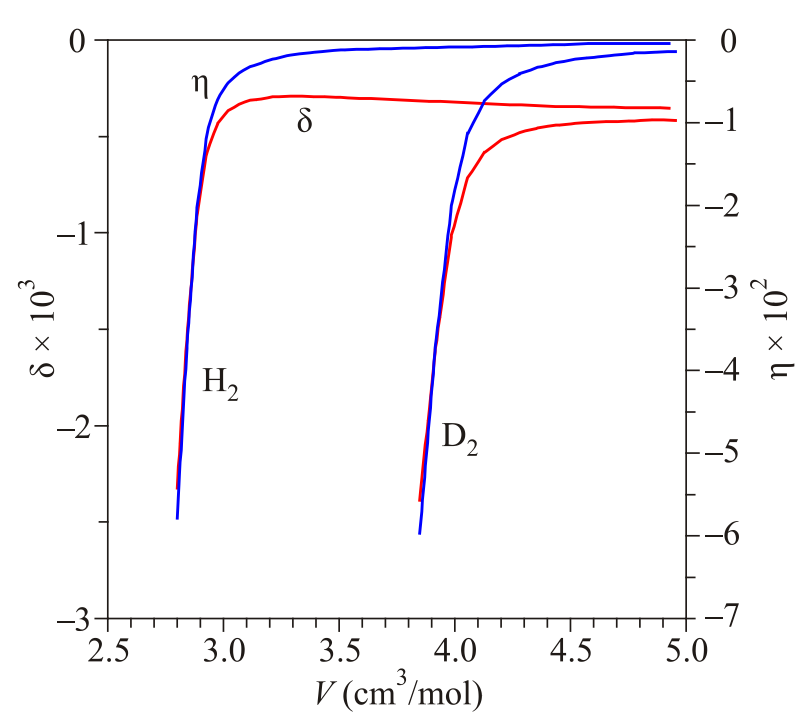

Fig. 7. (Color online) Lattice distortion parameter $\delta$ and orientational order parameter $\eta$ in parahydrogen and orthodeuterium as a function of molar volume [47].

have measured low-frequency Raman spectra at low temperature for the pressure range up to the I-II phase transition and used these spectra to estimate the crystal-field parameter $\varepsilon_{2 c}$. Assuming that only the second-order crystal field is responsible for the splitting of the roton triplet band $S_{0}(0)$ and get that $\left|\varepsilon_{2 c}\right| \sim 1 \mathrm{~cm}^{-1}$ and thus $|\delta| \sim 10^{-3}-10^{-4}$ in accord with theoretical data shown in Fig. 7.

There were numerous structural studies of $n-\mathrm{H}_{2}$ and $n-\mathrm{D}_{2}$ [3,31,48-51]. Synchrotron single-crystal x-ray diffraction measurements of $n-\mathrm{H}_{2}$ [31,48] and $n-\mathrm{D}_{2}$ [48] up to megabar pressures at room temperature revealed an approximately linear decrease of the $c$ / $a$ ratio with increasing pressure (Fig. 8). No isotope effect in the pressure dependence of the $c$ / $a$ ratio was found.

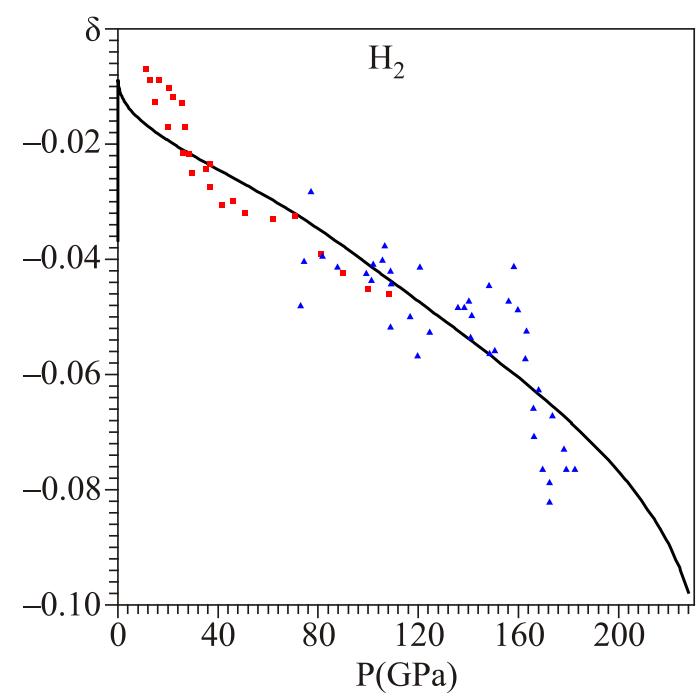

Fig. 8. (Color online) Lattice distortion parameter in normal ortho-para mixture of solid hydrogens. Theory: solid line [47]; experiment: red squares — data from Ref. 48, blue triangles — data from Ref. 31. 
For a single $J=1$ molecule in a lattice of $J=0$ molecules there is an additional contribution to the ground-state energy arising from the polarization of the surrounding $J=0$ molecules by the the electric quadrupole field of the $J=1$ molecule. The polarization energy due to the interaction of the quadrupole moment of the $J=1$ molecule with the induced dipole moments of the surrounding nearest neighboring $J=0$ molecules equal to

$$
\varepsilon_{1}=-18 \alpha Q^{2} R^{-8},
$$

where $\alpha$ is the polarizability of the $J=0$ molecules and $Q$ is the quadrupole moment of the $J=1$ molecule, and $R$ is the intermolecular distance.

If the crystal shows a homogeneous deviation from the ideal hcp structure, the polarization energy contains a crystal-field term [2]

$$
V_{c}=\varepsilon_{2 c} \sqrt{\frac{4 \pi}{5}} Y_{20}(\mathbf{\Omega}),
$$

where $\boldsymbol{\Omega}$ specifies the orientation of the $J=1$ molecule with

$$
\varepsilon_{2 c}=-\frac{24}{7} \varepsilon_{1} \delta_{c}
$$

The splitting of the $J=1$ level in the crystal field $V_{c}$ is given by

$$
\Delta_{c}=E( \pm)-E(0)=\frac{3}{5}\left|\varepsilon_{2 c}\right|,
$$

where $E(M)$ is the energy of the state $J=1, J_{Z}=M$ with $z$ along the $c$ axis. The positive sign of $\Delta_{c}$ implies that the singlet state $J_{z}=0$ is the ground state of the triplet. Due to this splitting the ground-state energy is brought down by $2\left|\varepsilon_{2 c}\right| / 5$. Thus, the gain in the anisotropic interaction contribution to the zero-point energy is linear in the lattice distortion parameter $\delta$. At the same time, this distortion increases the contribution to the ground-state energy from the isotropic part of the intermolecular interaction. This contribution is quadratic in the lattice distortion parameter $\delta$ [52]. The loss in the isotropic part and the gain in the anisotropic part of the ground-state energy determines the lattice distortion parameter at the given molar volume. One cannot obtain a reliable value of $\delta$ from Eqs. (9)-(12) because there are other contributions to the splitting Eq. (12) [2]. Assuming that the volume dependence of the polarization energy holds the same volume dependence as in Eq. (9) in spite of these contributions, we will get the pressure dependence of the lattice distortion parameter shown in Fig. 8. When this result is compared with that for $p-\mathrm{H}_{2}$ (Fig. 7) it is seen that the presence of $J=1$ molecules increases the hcp lattice distortion by two order of magnitude.
3.3. Experimental determination of the crystal field splitting of isolated $J=1$ impurities in solid parahydrogen

An isolated $J=1$ orthohydrogen substitutional molecule in the pure $J=0$ hcp crystal lattice is an ideal system which has been studied extensively both theoretically and experimentally [2]. The heat capacity anomaly in the $J=0$ solid hydrogen samples containing small concentrations of $J=1$ impurity molecules has been found by Mendesson, Ruhemann and Simon as early as in 1931 [53] and explained by Schaefer [54] in 1939 by the splitting $\Delta_{c}$ of the triplet level of the $J=1$ impurity molecules (Eq. (12)). In succeeding years this splitting has been studying in numerous heat capacity $[57,58]$ and NMR $[22,63-65]$ measurements, and theoretical works [55,56,59-61,66,67].

The first direct measurements of the crystal field splitting of the $J=1$ sublevels were performed by Dickson, Bayers and Oka [68] who observed the fine structure of the infrared $Q_{3}(0)$ second overtone transition $(v=3 \leftarrow 0$, $J=0 \leftarrow 0$ ) at $11758 \mathrm{~cm}^{-1}$ in parahydrogen crystals containing approximately $0.1 \%$ orthohydrogen. The transition is caused by the dipole moments induced in $J=0 \mathrm{H}_{2}$ due to the quadrupolar electric field of the $J=1 \mathrm{H}_{2}$.

Three main sets of features were observed: the stronger sets separated by nearly $0.5 \mathrm{~cm}^{-1}$ which arise from nearest neighbor pairs of $J=1 \mathrm{H}_{2}$ and $J=0 \mathrm{H}_{2}$, while the weaker set near the center is due to next nearest neighbor pairs. The large splitting of $0.5 \mathrm{~cm}^{-1}$ corresponds to that of the $J=1 \mathrm{H}_{2}$ next to the vibrationally excited $J=0 \mathrm{H}_{2}$ : the vibrational excitation breaks the $D_{3 h}$ symmetry of the crystal and the splitting is greatly enhanced since the lattice sum for nearest neighbors no longer vanishes. The breakdown of symmetry also removes the degeneracy of the $M= \pm 1$ levels; this together with the slight difference in energy for in plane and out of plane pairs leads to the intricate structure of each set.

The small $J=1 \mathrm{H}_{2}$ splitting without vibrational excitation for $J=0 \mathrm{H}_{2}$ is shown in Fig. 9 where the set of transitions near $11758.39 \mathrm{~cm}^{-1}$ is given with an expanded frequency scale for the polarization of the laser radiation both parallel and perpendicular to the crystalline $c$ axis. Alto-

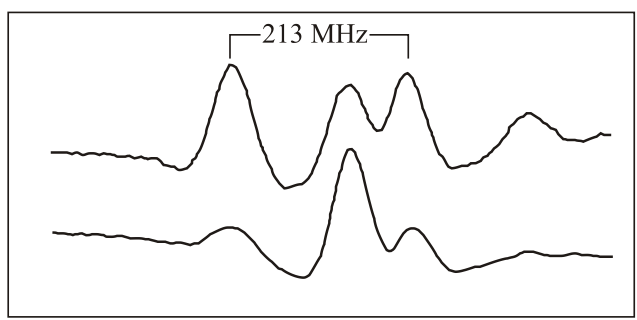

Fig. 9. The low-frequency set of transitions for both parallel (upper) and perpendicular (lower) polarizations. The first and third lowest frequency transitions are due to out-of-plane nearest neighbor $J=1 / J=0$ hydrogen molecules while the second and fourth are due to in-plane neighbors [68]. 
gether eight such pairs of transitions are observed and led to the value $\Delta_{C}=(0.0102+0.0003) \mathrm{K}$ and to the observation that the $M= \pm 1$ levels are higher in energy than is the $M=0$ level. This value agrees with the results of Schweizer et al. [64] within their quoted uncertainty and is close to the theoretical estimate of Raich and Kanney [66].

\subsection{HCP lattice distortion in solid HD}

The principal difference between the HD molecule and its homonuclear isotopic analog is that the center of mass is considerably shifted relative to the center of electron density distribution. A free HD molecule rotates around its center-of-mass, which is separated from the midpoint between the two nuclei, the center-of-charge, by a distance $1 / 6 r_{e}$, where $r_{e}$ is the internuclear distance $\left(r_{e}=0.74116 \AA\right)$. Due to such mismatch the heteronuclear solid isotopes HD, HT, and DT have some properties that distinguish them from the homonuclear species. The most remarkable case the reentrant BSP phase transition in solid HD will be discussed in Sec. 4. In this section we discuss the effect of the molecular asymmetry on the axial ratio of the hcp lattice parameters $c / a$.

Structure data obtained using x-ray diffraction [69] (Fig. 10) gave an unexpected result: $c / a=1.618 \pm 0.003$ at the zero-temperature limit while for $p-\mathrm{H}_{2} \mathrm{x}$-ray diffraction measurements by Krupskii et al. [43] gave the ideal value $1.633 \pm 0.001$. Surprisingly, the $c / a$ ratio showed different temperature behavior for different species: whereas in both normal and para $\mathrm{H}_{2}$ the $c / a$ ratio slightly decreased [69] upon warming, the $c / a$ ratio in HD grew with temperature, reaching the value $1.623 \pm 0.005$ at $17 \mathrm{~K}$. The authors qualitatively explained the found effect by the different virtual contributions of the excited rotational states into the molecular ground rotational state of the molecule. Theory of the effect developed recently by Strzhemechny and Hemley [74] explains how differences in the rotational dynamics affect the value of the $c / a$ ratio at low temperatures. Their approach is based on the following equation:

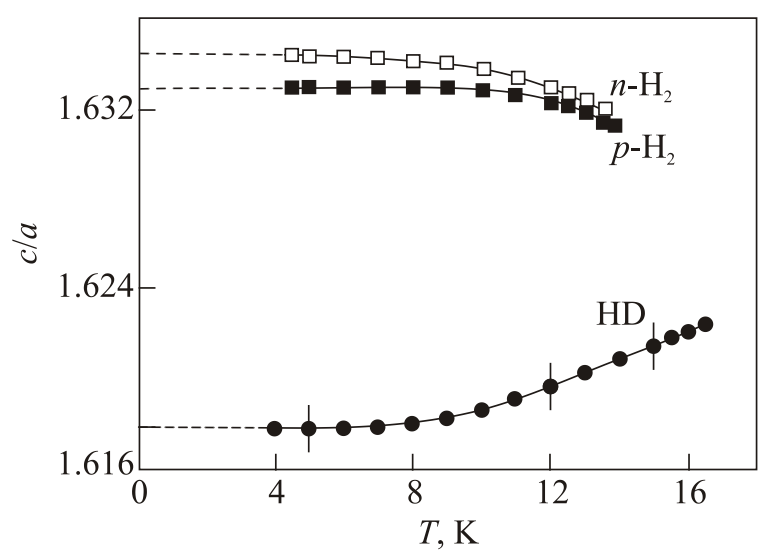

Fig. 10. Temperature dependencies of the axial ratio of the hcp lattice parameters $c / a$ for deuterohydrogen [69], parahydrogen [43], and normal hydrogen [70]. After Ref. 69.

$$
\frac{\partial}{\partial \delta}\left[\Delta E_{\mathrm{el}}(\delta)+\Delta E_{\mathrm{rot}}(\delta)\right]=0
$$

where $E_{\text {el }}$ and $E_{\text {rot }}$ are the densities of the elastic and ground-state rotational energies, respectively, as functions of the deviation $\delta$. While the loss in the elastic energy $\Delta E_{\mathrm{el}}(\delta)$ is proportional to $\delta^{2}$, the gain in the rotational energy $\Delta E_{\text {rot }}(\delta)$ due to the quantum self-polarization effect calculated in the second perturbation order in the anisotropic interactions is linear in $\delta$. Taking both contributions into consideration one gets a nonzero deviation $\delta$. The final estimate for $\delta$ in solid HD is

$$
\delta(\mathrm{HD})=-(0.61 \pm 0.08) \cdot 10^{-3}
$$

the deviation $\delta$ in HD is also negative and approximately 50 times larger in magnitude than in $\mathrm{H}_{2}$ or $\mathrm{D}_{2}$ at zero pressure (see Fig. 7). Though the above value is by a factor of approximately 20 smaller compared with what was determined experimentally [69], the trend is correct.

\section{Broken symmetry phase transition in solid HD and $o-p$ mixtures}

\subsection{Introduction}

To a very good approximation, the electron density distribution in the $\mathrm{H}_{2}$ and $\mathrm{HD}$ molecules are the same, but in the HD molecule the center-of-charge does not coincide with the center-of-mass. Since the molecule rotates around its center-of-mass but the intermolecular interaction is related to the center-of-charge molecule, rotations of the molecules are accompanied by translational displacements of the center-of-mass. Thus, rotation and translation of the molecule are coupled dynamically. As a result of such offcenter rotation, an additional Heisenberg-like terms appear in the anisotropic part of the intermolecular potential [2,71].

Evidence for differences in properties of symmetric and asymmetric hydrogens resulted from the rotational-translational coupling in the condensed state has been reported since the sixties of the last century [72].

The heteronuclear hydrogen molecules have nonzero static electric dipole moments. The experimental value of the HD dipole moment, as derived from pure rotational spectra of gaseous $\mathrm{HD}$, is $p=5.85 \cdot 10^{-4} \mathrm{D}$ [73]. Because of the nonequivalency of the ends of the HD molecule, there is an additional dynamics in solid HD in comparison with homonuclear isotopes $\mathrm{H}_{2}$ and $\mathrm{D}_{2}$, namely, end-to-end reorientational dynamics [2]. In principle, by ordering the dipole electric moments it is possible to minimize the dipole-dipole interaction energy. Thermodynamics and kinetics of the dipole ordering have been discussed in literature in connection with the problem of the dipolar ordering and zero-temperature entropy in solid CO, a diatomic possessing in addition to a large quadrupole moment a small permanent dipole moment $\sim 0.1 \mathrm{D}$, two orders of magnitude larger than HD [75]. In the case of solid CO the tem- 
perature of the hypotetical dipole ordering phase transition is about $5 \mathrm{~K}$ [3], but the transition is frozen because the molecules are ordered by much stronger quadrupolequadrupole interaction at $T \sim 40 \mathrm{~K}$. The qualitative discussion of the possible dipole ordering in solid HD was done by Chijoke and Silvera [76]. The characteristic dipolar energy of an HD molecule in the field of the nearest neighbors $E_{d} \sim z p^{2} / R^{3}$ (where $z$ is the number of nearest neighbors and $R_{n n}$ is the nearest neighbor distance) gives for the transition temperature an estimate $\sim 1 \mathrm{mK}$. Such transition cannot take place in the quantum phase I since all the molecules are in the $J=0$ state and the expectation value of the dipole moment in this state is zero. It has been speculated by the authors of Ref. 76 that the conditions to observe the dipole ordering transition in $\mathrm{HD}$ are much more favorable at high pressure. They estimated that at $P \sim 150 \mathrm{GPa}$ the dipole moment induced in the molecule by the quadrupole moment of neighboring molecule in $J=1$ state is $\sim 24$ times larger than the permanent dipole moment and this could increase the interaction energy to tens of K. Summarizing results of the IR study Ref. 76 the authors stated that in their low-temperature spectra they "found no indication of a transition of electric dipole ordering”. We think that even though their considerations about the strong enhancement of the dipolar interaction at high pressure are correct, the dipolar ordering in HD is inobservable due to the same reason why it cannot be realized in solid CO: dipole end-to-end reorientations are frozen in a strong quadrupolar field.

The BSP transitions in the even- $J$ species (parahydrogen and orthodeuterium) and all- $J$ system (HD) provide an example of quantum phase transitions where the orientational ordering (OO) is realized by a competition between the potential and kinetic energies of the system as opposed to the classical phase transitions in the $J$-odd species (orthohydrogen and paradeuterium) where the $\mathrm{OO}$ is realized by a competition between the potential energy and the entropy contribution to the free energy of the system. As a result, strong differences are observed in the OO properties of odd- $J$, even- $J$, and all- $J$ (e.g., HD) systems. The odd- $J$ systems order at any pressure at low temperature, while the molecules in the even- and all- $J$ systems at low pressure are in the $J=0$ quantum state and thus are spherical entities. The $\mathrm{OO}$ in the even- and all- $J$ systems is realized by increasing the pressure, that is by increasing the potential energy of the molecules. By analogy with quantum melting of solid helium the quantum orientational disordering in even- and all- $J$ solid hydrogens was taken the title Quantum Orientational Melting (QOM) [80-83].

The phase diagram of all- $J$ systems possesses a surprising anomalous feature: there is a pressure range $P_{m}<P<P_{0}$ ( $P_{0}$ is the ordering pressure at $T=0, P_{m}$ is a minimal ordering pressure at the transition line) where the disordered phase is reentrant (see Fig. 11). At $T=0$ in this pressure range the system is in the disordered state but on heating we cross the phase transition line and get to the ordered phase. By increasing the temperature further at constant $P$ we get once again to the disordered phase. And conversely, if we start from the ordered state inside the pressure range $P_{m}<P<P_{0}$, we can get from the ordered to the disordered state by two ways: either by heating or by cooling! In the former case the order is destroyed by thermal fluctuations, in the latter case the order is destroyed by quantum fluctuations. This reentrant behavior was first predicted for the all- $J$ system by mean-field calculations $[80,81]$ and found experimentally in HD by Silvera's group [78].

As was shown in Refs. 80, 81, there is a very close analogy between the behavior of the melting line in solid ${ }^{3} \mathrm{He}$ and the BSP transition in HD. As known, at the lowtemperature section of the melting line of ${ }^{3} \mathrm{He}$, the entropy of liquid is smaller than that of the solid (Pomeranchuk effect). As a result, there is a minimum in the pressuretemperature dependence of the melting line in ${ }^{3} \mathrm{He}-$ the melting transition in ${ }^{3} \mathrm{He}$ is reentrant. It was shown that the reentrance in the BSP transition line in all- $J$ rotor systems has also entropy-driven origin [80,81].

Path integral Monte Carlo (PIMC) technique for studying the reentrant behavior in solid HD was applied in Ref. 84. The system of asymmetric rotors was considered with centers fixed on fcc lattice with the EQQ interaction as the anisotropic interaction potential. The results were only of qualitative value. Recently a more accurate approach was implemented by Crespo et al. [85] where the authors applied to solid HD at high densities PIMC within the constant pressure ensemble. They considered both the translational and rotational degrees of freedom and different sources for the anisotropic interaction potential. The HD phase line was calculated for both fcc and hcp lattices, displaying the reentrant behavior in both cases. Good agreement with the experimental data was obtained in the case of the hcp lattice, including the minimum pressure $\left(P_{m}\right)$ where the BSP transition occurs $\left(P_{m}=56 \mathrm{GPa}\right)$ and temperature at the minimum point $\left(T_{m}=25 \mathrm{~K}\right)$ to be compared with experiment: $P_{m}=53 \mathrm{GPa}, T_{m}=30 \mathrm{~K}$ [78].

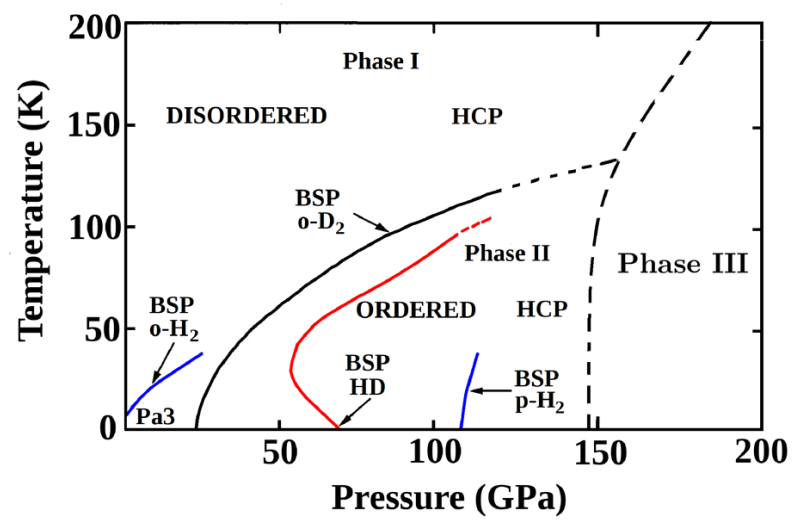

Fig. 11. Schematic experimental phase diagram for $\mathrm{H}_{2}$, ortho- $\mathrm{D}_{2}$ and HD from data reported in Refs. 77-79. 


\subsection{Experimental results}

Experimentally, "Remarkable high pressure phase line of orientational order in solid hydrogen deuteride” (as was the title of the first publication), was first found by means of Raman scattering by Silvera's group [78] and then studied to higher pressure by using near infrared spectroscopy [76]. It was observed that in HD the BSP transition pressure first decreases and then increases with increasing temperature, a very unusual reentrant behavior, different from the homonuclear isotopes $\mathrm{H}_{2}$ and $\mathrm{D}_{2}$. In this Raman study the authors measured both the Raman active rotons and vibrons in HD to pressures of $120 \mathrm{GPa}$ and temperatures as low as $3.5 \mathrm{~K}$ (Fig. 12(a) [78]). The HD vibron frequency shifts substantially with changing pressure at the phase line and due to the sharpness of the vibron the detection of this discontinuity was used as a method for determining the phase line. In Fig. 12(b) the discontinuity is seen on the $64 \mathrm{GPa}$ curve. The intensity of the peak shifts from one vibron to the other when the phase line is crossed. At the lowest temperature of this experiment $(3 \mathrm{~K})$ a critical pressure $P_{C}=68.3 \mathrm{GPa}$ GPa was obtained. The critical pressure at $T=0$ was estimated by extrapolation to be $P_{0}=(69.0 \pm 2) \mathrm{GPa}$, lying between the values for $\mathrm{H}_{2}$ and $\mathrm{D}_{2}$ solids.

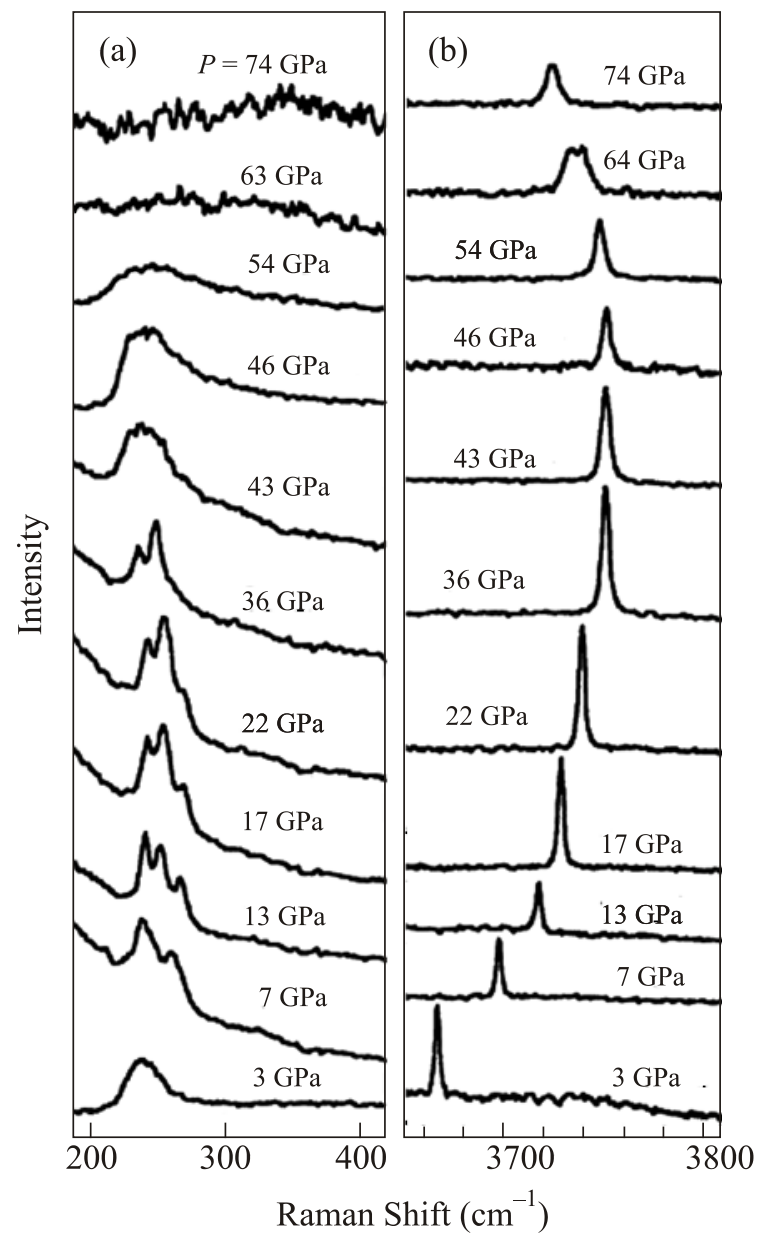

Fig. 12. Raman spectra of HD for several pressures with temperature 5-7 K. (a) Rotational spectra, (b) vibron spectra (after Ref. 78).
As the Raman line broadened with increasing pressure and the shift of the line became too small to detect the BSP, the work Ref. 78 was limited in pressure by 74 GPa. Another technique that can be used to study this phase transition at low temperatures is the infrared (IR) absorption. In Ref. 76 the authors reported the first infrared absorption measurements on HD to pressures of $159 \mathrm{GPa}$. In Fig. 13 the IR spectra are shown in the region of the firstorder band for pressures between 11 and $159 \mathrm{GPa}$ at $5 \mathrm{~K}$. These spectra correspond to the $v(0 \rightarrow 1)$ vibron transition. The $0 \rightarrow 1$ vibrons of HD in hcp solid are symmetry forbidden for IR absorption at low temperature when the thermal occupation of the $J=1$ level is close to zero. The transition to the BSP phase was determined by the appearance of two strong and relatively sharp absorption peaks with linewidths of 18 and $40 \mathrm{~cm}^{-1}$ (see the curve at $77 \mathrm{GPa}$ in Fig. 13), as well as very weak third peak, indicated by arrows in Fig. 13. These lines were interpreted as IRallowed $v(0 \rightarrow 1)$ vibrons in the BSP phase. The reentrant behavior of the BSP phase was observed by the appearance and disappearance of these lines as the temperature was raised at a fixed pressure of $56 \mathrm{GPa}$. In the ultra-highpressure region (159 GPa) it was found that the system undergoes a transition from the BSP to the phase III. At pressures of $(157 \pm 3) \mathrm{GPa}$ it was observed the appearance of a new spectral feature in the IR spectrum, a new peak at $3890 \mathrm{~cm}^{-1}$ (see the arrow in $159 \mathrm{GPa}$ in Fig. 13). This is

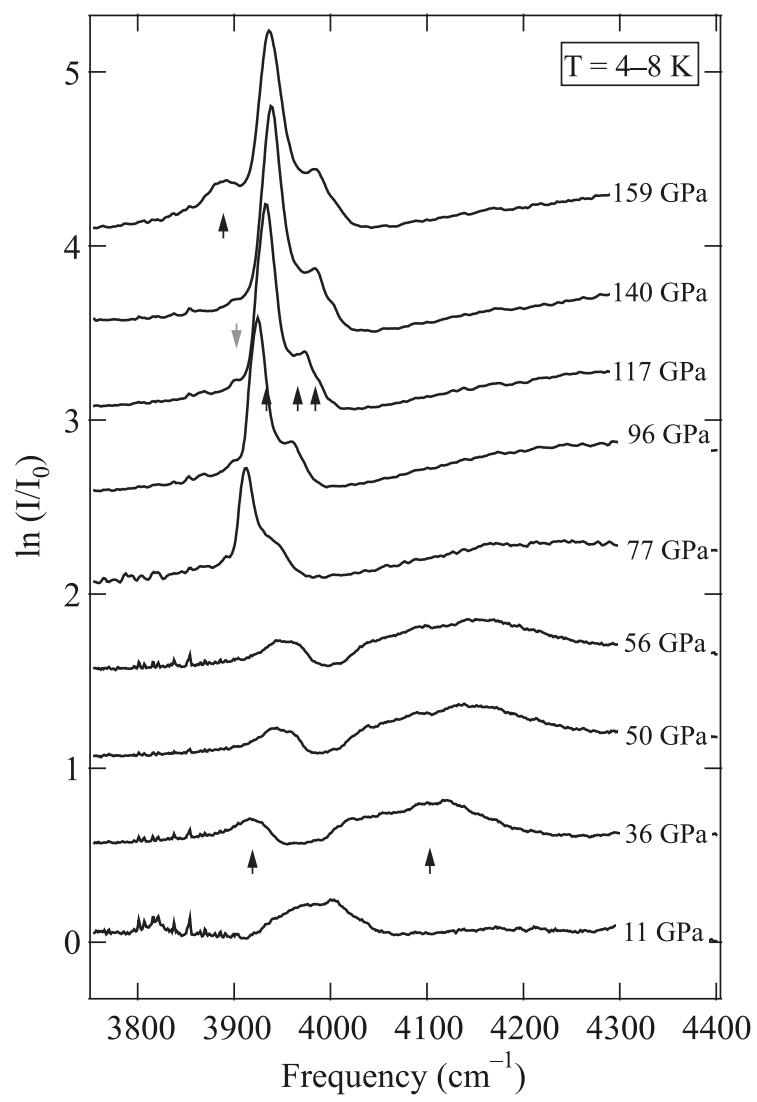

Fig. 13. Absorption spectra for several pressures, collected at temperatures of 4-8 K (after Ref. 78). 


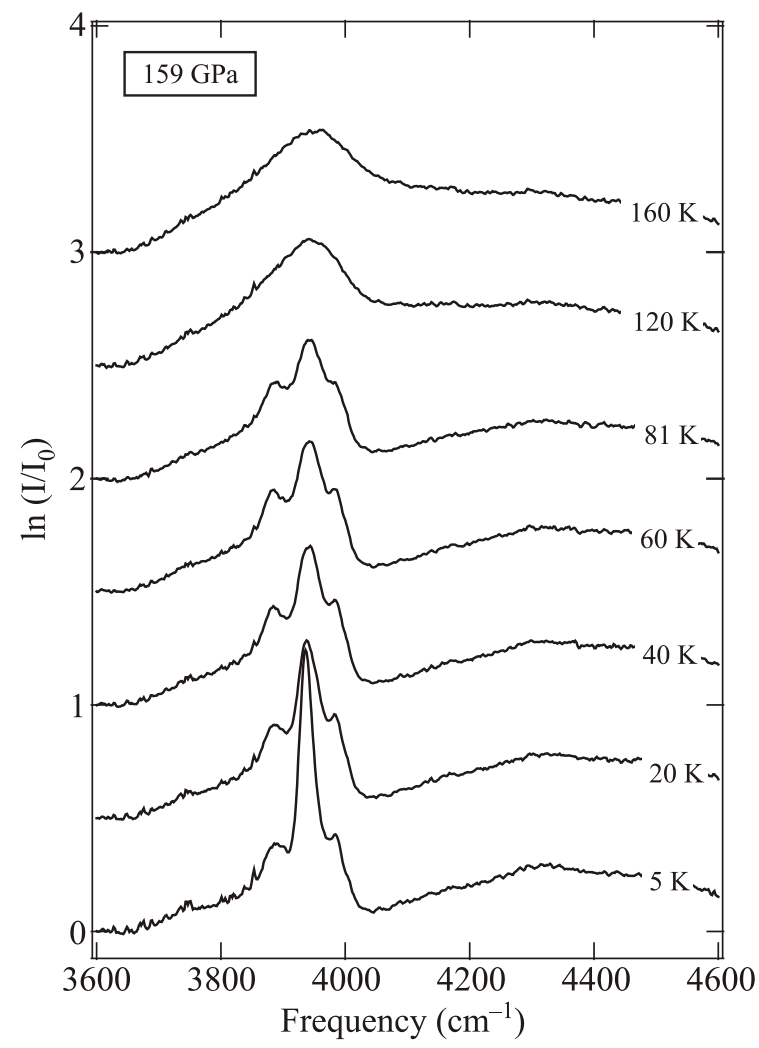

Fig. 14. Absorption spectra collected at 159 GPa for several temperatures in the range between 5-160 K (after Ref. 76).

the same pressure range and spectral response for the onset of the phase III in $\mathrm{H}_{2}$ [86] and $\mathrm{D}_{2}$ [87]. As a result, these data were were interpreted as a first observation of phase III in HD [76]. In Fig. 14 the absorption spectra are shown collected at $159 \mathrm{GPa}$ for several temperatures. As can be seen from Fig. 14, the intensity of the peaks corresponding

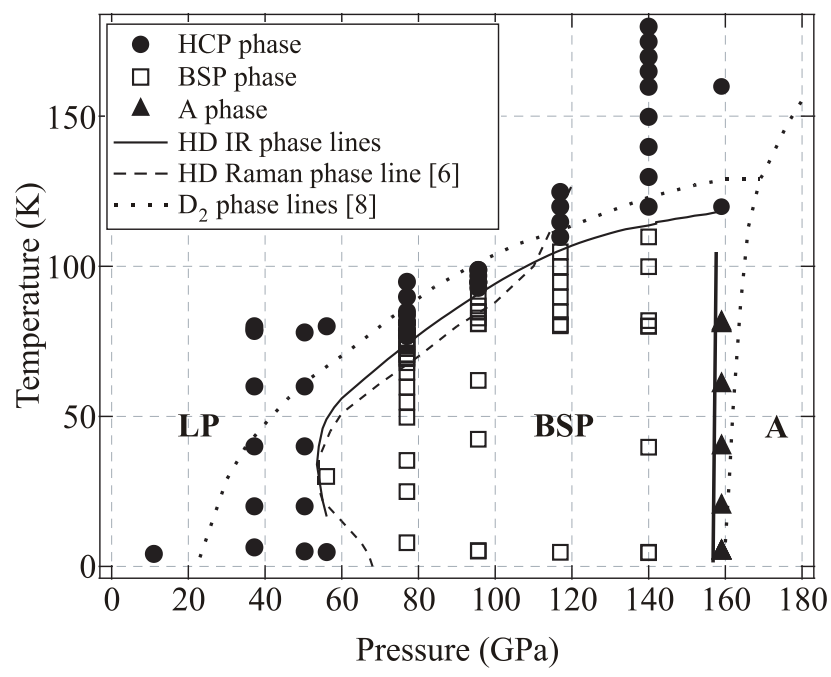

Fig. 15. Phase diagram of HD, the phase lines determined in Ref. 76 are shown as solid lines and the phase line determined earlier by Raman measurements as dashed lines [78]. The phase lines of $\mathrm{D}_{2}$ (dotted lines) [88] are also shown for comparison (after Ref. 76). to both the phase III and BSP decreases as temperature is raised becoming at $120 \mathrm{~K}$ the characteristic spectrum of the hcp disordered phase. The phase transition line determined from these methods are shown in Fig. 15.

\subsection{Theoretical studies}

\subsubsection{Quantum orientational melting in all-J system}

The BSP transition in solid hydrogen, is a realistic example of Quantum Orientational Melting (QOM) that can occur at zero temperature. This quantum phase transition is driven by a competition between the potential energy, which tends to order the system, and the kinetic energy, which tends to delocalize the system. Early mean field theoretical models have shown that this type of transition can be observed in a system of rotors under appropriate conditions [80-82]. The mean field approximation considers a system of $N$ linear quantum rotors described by the Hamiltonian [80-82]

$$
\mathcal{H}=B_{\text {rot }} \sum_{i}^{N} \hat{L}^{2}-U \eta \sum_{i}^{N} Y_{20}\left(\Omega_{i}\right)+U N \eta^{2} / 2,
$$

where $\hat{L}$ is the angular momentum operator, $U$ is a molecular field constant $(U \sim P)$, $B_{\text {rot }}$ is the rotational constant $\eta=\sqrt{4 \pi / 5},\left\langle Y_{20}\left(\Omega_{i}\right)\right\rangle$ is the order parameter, and $\langle\ldots\rangle$ denotes thermodynamic averaging with the Hamiltonian (15).

The computational scheme used to find the spectrum of the Hamiltonian (15) is given in Refs. 80-82, 90. Figure 16 shows the energy spectrum for a linear rotor in the field $-V P_{2}(\cos (\vartheta))$ where $V=U \eta$. As can be seen from this figure, in the disordered phase $(\eta=0)$ the gap $\Delta$ between the ground state and the first exited triplet state is $\Delta=2 B_{\text {rot }}$. In the ordered phase of an all- $J$ system, instead, this degeneracy is splitted into a singlet and a doublet so that $\Delta$ decreases as increasing $\eta$ (for constant $U$ ). In the the strong interaction limit the ground state becomes a doublet. This reduction of the gap allows the occupation of this level even at low temperatures and as a result there is an additional contribution to the entropy, of the order of $k_{B} \ln 2$ per a molecule where $k_{B}$ is the Boltzmann constant, that stabilizes the ordered phase at low temperatures.

The previous reasoning was confirmed by the calculations of the entropy and order parameter with temperature. Figure 17 shows the temperature dependence of the entropy of both the one corresponding to the free rotor and that of the $\mathrm{OO}$ state for different values of $U / B$ $(11.5<U / B<15.0)$. As can be seen, in the low- $T$ region the entropy of the OO phase is larger than that of the disordered one. The inset shows the temperature dependence of the difference in entropy $\left(\Delta S=S-S_{0}\right.$ ) between the ordered, $S$, and the disordered, $S_{0}$, phases for $U / B \approx 12.9$.

The free energy of the system as a function of the order parameter $\mathcal{F}(\eta)$ was calculated using the obtained spec- 


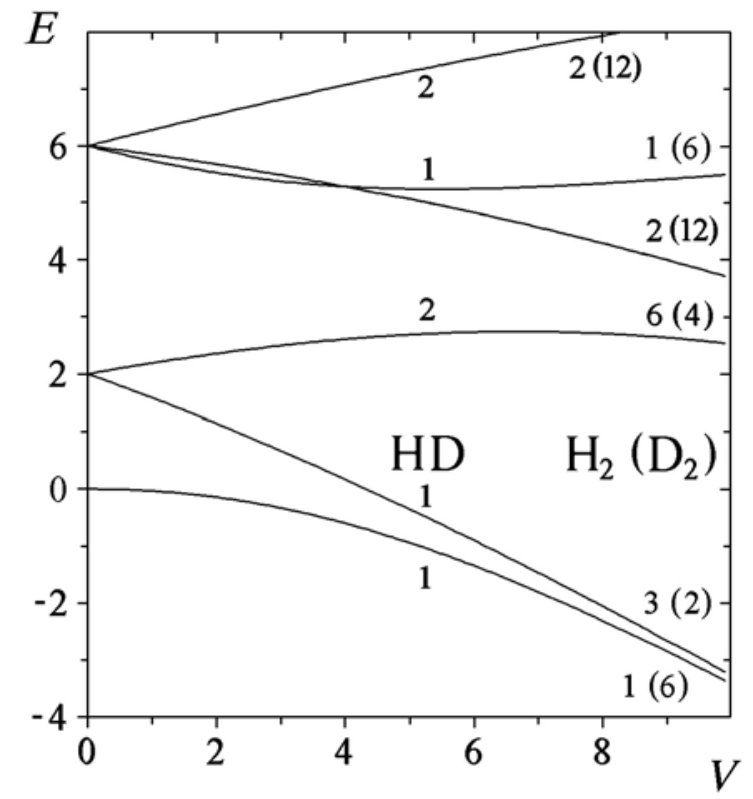

Fig. 16. Energy spectrum of a hindered linear rotor in the field $V P_{2}(\cos (\vartheta)) . E$ and $V$ are given in units of the rotational constant $B_{\text {rot }}$. The numbers at the curves indicate the degeneracy of the even- and odd- $J$ energy levels for $\mathrm{H}_{2}, \mathrm{D}_{2}$, and $\mathrm{HD}$, respectively, taking into account nuclear spin degeneracy (after Refs. 80, 81, 89).

trum (Fig. 16), and from the condition $\partial \mathcal{F} / \partial \eta=0$ the order parameter was found as a function of temperature.

Let us consider the situation for $P_{m}<P<P_{0}$ where ordering at zero temperature is absent. As one can see from Fig. 18, there is a range of pressures where the ordered

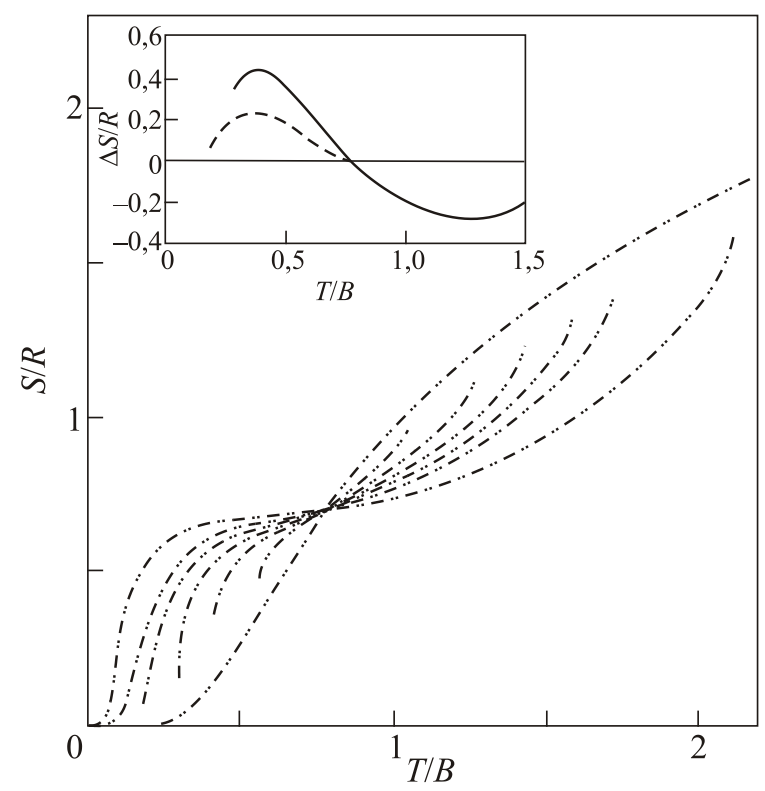

Fig. 17. Temperature dependence of the entropy for free rotor and for the orientational ordered state of an all- $J$ system in the field $-U \eta P_{2}(\cos (\vartheta))$ at different values of $U$ (dot-dashed lines). The solid line in the inset shows the entropy difference between the ordered and disordered phases for $U / B \approx 12.9$ (after Ref. 81).

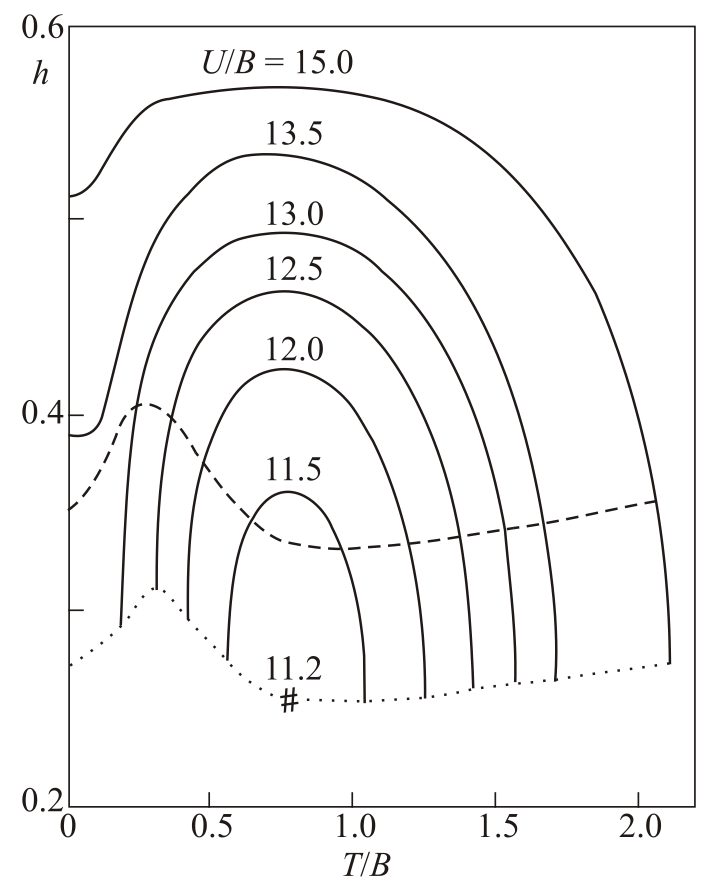

Fig. 18. Temperature dependence of the order parameter $\eta$ of a system of all- $J$ rotors. The dash curve is the line of equilibrium phase transitions, the dotted curve is the loci of absolute instability of the orientationally ordered state (after Ref. 81).

phase emerges with increasing temperature. Within this range of pressures the system undergoes two first-order phase transitions with increasing temperature, viz., the low-temperature one from disordered to ordered state and the hightemperature one from ordered to disordered state. The former is of a quantum nature, QOM, the latter is the common orientational disordering. This behavior has the following explanation. At $T=0$ the only ordering factor is the potential energy; as temperature increases the singlet of the splitted triplet start to get occupied increasing the contribution of the entropy term ( $T \Delta S$ ) of the free energy, when the sum of the contribution coming from the potential energy and the term $T \Delta S$ became bigger than the contribution from the kinetic energy a phase transition to the ordered

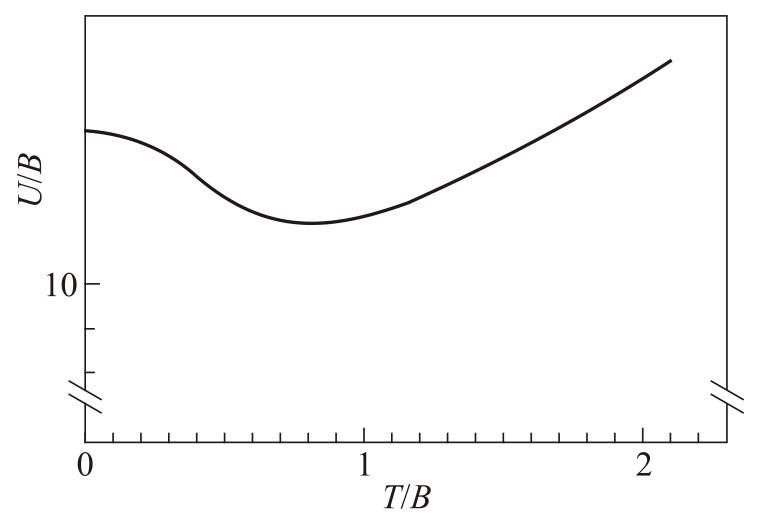

Fig. 19. The $U-T$ phase diagram for a system of interacting rotors under the field $-V P_{2}(\cos (\theta))$ where $V=U \eta$ (after Refs. 80, 81). 
phase takes place. When temperature grows further higher levels start to get occupied, as a consequence $\Delta S$ arrive to a maximum and begin to decrease till the entropy of the disordered state became grater than the ordered one and a second phase transition to the disordered phase occurs. As a result the phase diagram of this system became reentrant as shown in Fig. 19 where the $U-T$ phase diagram for systems of interacting rotors is plotted [80,81].

\subsubsection{The reentrant behavior from numerical simulations}

The first attempt to go beyond mean field approach and study the reentrant behavior in solid HD by using standard numerical simulations was made in Ref. 84. In this work the Path Integral Monte Carlo technique [91] (PIMC) was employed using two main approximations: (i) the authors consider asymmetric rotors with centers fixed at sites of fcc lattice instead of hcp, and (ii) their anisotropic interaction potential was limited by the pure EQQ interaction:

$$
B_{224}\left(R_{n n}\right)=\frac{\Gamma_{0}\left(R_{n n}^{0}\right)^{5}}{(4 \pi)^{3 / 2}}\left(\frac{1}{R_{n n}}\right)^{5},
$$

where $R_{n n}^{0}$ is the nearest neighbor distance at zero temperature. Even when the EQQ interaction is the leading term of the pair interaction potential its selection is essential for getting the right transition pressure [85]. It results that the anisotropic interaction potential used in this work [84] was too soft [85] and as a consequence the minimum transition pressure $P_{m}$ obtained was small $P_{m} \approx 10 \mathrm{GPa}$ when compared with the experimental value $P_{m}=53 \mathrm{GPa}$ (see Fig. 20). Recently a more accurate approach was implemented by Crespo et al. in Ref. 85. In this paper, the authors model the HD solid, using the constant pressure PIMC (CP-PIMC) formalism. The HD solid was simulated as an assembly of rigid molecules, positioned on both fcc or hcp lattices and having rotational and translational de-

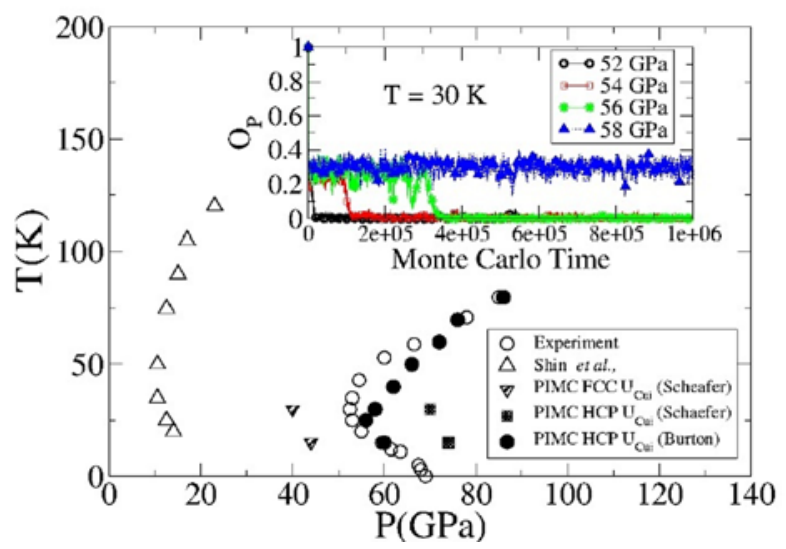

Fig. 20. The temperature-pressure phase diagram of solid HD. The open circles are the experimental data [78]. The up open triangles are the constant volume PIMC results reported by Shin et al. [84]. All other symbols are constant pressure PIMC results obtained by Crespo et al. [85]. grees of freedom. The pair interaction potential used was essentially that of Cui et al. [92]

$$
U_{\text {Cui }}=\sum_{i<j} U_{\text {iso }}\left(R_{i j}\right)+\kappa \sum_{i<j} U_{\text {ani }}^{\text {pair }}\left(\Omega_{i}, \Omega_{j}, \mathbf{R}_{i j}\right),
$$

where $U_{\text {iso }}\left(R_{i j}\right)$ is the isotropic component, $\mathbf{R}_{i j}$ is the vector connecting the center points of two molecules, $\Omega_{i}=\left(\theta_{i}, \phi_{i}\right)$, is a vector containing the inclination and azimuthal angles $\left(\theta_{i}, \phi_{i}\right)$ in spherical coordinates and $\kappa$ is a rescaling factor. Two sources for the anisotropic coefficients $U_{\text {ani }}^{\text {pair }}$ were considered: first Schaefer et al. [93], denoted as $U_{\text {Cui }}^{\text {Schaefer }}$; second, Burton et al. [94], denoted by $U_{\mathrm{Cui}}^{\text {Burton }}$. In order to determine the BSP line of HD it is necessary to define an appropriate order parameter to measure and monitor the angular order. Suppose we know the structure of the ordered phase; then it is possible to define $N$ unit vectors $\mathbf{u}_{i}$ corresponding to the orientation of molecule on all sites $i=1, \ldots, N$ and define the order parameter:

$$
O_{p}=\left[\sum_{m=1}^{M} \sum_{i=1}^{N} \frac{1}{2 M N}\left(3 \cos ^{2}\left(\mathbf{n}_{i, m} \cdot \mathbf{u}_{i}\right)-1\right)\right]^{2},
$$

where $M$ is the total number of Trotter slices [91], $\mathbf{n}_{i, m}$ labels the orientation of the molecule $i$ in the Trotter slice $m$ for a given configuration visited with the PIMC algorithm. This order parameter measures the extent of ordering (and by difference, its deviation) relative to a given orientational structure defined by the set $\left\{\mathbf{u}_{i}\right\}$ and have been extensively used to study the BSP of solid hydrogens [84,85,92,95,96]. Nevertheless the order parameter in Eq. (18) suffers from an important limitation, namely: if the system reaches an ordered structure different from the reference one associated to the chosen set $\left\{\mathbf{u}_{i}\right\}$, still $O_{p} \approx 0$. Thus, the condition $O_{p}=0$ is not sufficient to ensure that the system is in an orientationally disordered phase. To address this issue further, a second order parameter defined as

$$
\left\langle O_{Q}\right\rangle=\frac{1}{N} \sum_{i=1}^{N} \frac{1}{6} \sum_{j, k=1}^{3}\left[\mathcal{Q}_{j k}^{\text {Total }}\right]^{2},
$$

can be monitored, where

$$
\mathcal{Q}_{j k}^{\text {Total }}=\frac{1}{T_{M C} M} \sum_{a=1}^{2 T_{M C}^{M}}\left(3 r_{j, a}^{i} r_{k, a}^{i}-r_{0}^{2} \delta_{j k}\right)
$$

with $T_{M C}$ is the MC time. $\mathcal{Q}_{j k}^{\text {Total }}$ is the quadrupolar moment of a system of $T_{M C} M$ molecules with a charge per atom of $\left(T_{M C} M\right)^{-1}$ [85]. If the molecules rotate in MC or Trotter time [91] (showing spherical symmetry), then $\mathcal{Q}_{j k}^{\text {Total }}=0$; if, on the contrary, the molecules are frozen in a quadrupolar configuration both in MC and Trotter time, then $\frac{1}{6} \sum_{j, k=1}^{3}\left[\mathcal{Q}_{j k}^{\text {Total }}\right]^{2}=1$. Thus $\left\langle O_{Q}\right\rangle$ signals quadrupolar 
order in a more general way than $\left\langle O_{p}\right\rangle$, which is restricted to a particular choice of $\left\{\mathbf{u}_{i}\right\}$.

The inset of Fig. 20 shows the order parameter $O_{p}$ as a function of MC time at four pressures: $52 \mathrm{GPa}$ (open circles), 54 GPa (open squares), 56 GPa (solid diamond) and $58 \mathrm{GPa}$ (solid up triangles). The abrupt change seen in the order parameter indicates the presence of a phase transition from a disordered phase to an ordered one, suggesting that $\mathrm{HD}$, at a temperature of $T=30 \mathrm{~K}$, orders at $\approx 58 \mathrm{GPa}$. Similar results were reported for the second order parameter $\mathcal{Q}_{j k}^{\text {Total }}$ [85]. Figure 20 shows the calculated temperature-pressure phase diagram of solid HD, along with the experimental transition line (open circles, from Ref. 78), also compared to theoretical phase diagrams obtained in previous calculations. The up open triangles are the constant volume PIMC results reported by Shin et al. [84]. The rest of the symbols are CP-PIMC results considering different lattices (fcc, hcp) and different interaction potential ( $U_{\text {Cui }}^{\text {Schaefer }}$ and $U_{\text {Cui }}^{\text {Burton }}$ ) obtained by Crespo et al. [85]. As can be seen the reentrant behavior for the HD phase line is obtained on both the realistic hcp and the fictitious fcc lattices, and for both choices of the anisotropic potentials. The minimum pressure at which the transition occurs is $P_{m} \approx 56 \mathrm{GPa}$ for the hcp lattice with the $U_{\mathrm{Cui}}^{\text {Burton }}$ potential, close to the experimental value of $P_{m}=53 \mathrm{GPa}$. Using alternatively $U_{\text {Cui }}^{\text {Schaefer }}$ yields instead $P_{m} \approx 70 \mathrm{GPa}$. The edge point temperature $T_{m}=25 \mathrm{~K}$ is also in good agreement with the experimental value of $T_{m}=30 \mathrm{~K}$. In general, the obtained BSP line with the $U_{\text {Cui }}^{\text {Burton }}$ potential is in good agreement with the experiment. These results show the crucial role played by the choice of the EQQ interaction in determining the BSP transition pressure. Interestingly, the BSP transition line for the fcc lattice is strongly shifted downwards by about $30 \mathrm{GPa}$, regardless of the potential used. In that case the BSP structure is $\mathrm{Pa}$, and the stronger tendency to order is evidently due to the lack of frustration in this structure. In other words, it is the angular frustration present in the hcp lattice, and the connected poorer relative stability of the $C 2 / c$ structure [85], that renders the BSP angular structure much more prone to melting than the Pa3.

To estimate the jump in entropy during this transition the slope of the BSP line can be used. In fact according to the Clapeyron equation $d P / d T=\Delta S / \Delta V$, therefore directly connected to the entropy jump $\Delta S$ between the low temperature quantum rotationally melted phase, and the higher temperature BSP solid phase. During this first order phase transition, the entropy jump vanish both at $T=0$ (because of the Nernst theorem), and at the reentrant edge point, where the entropies of both phases have the same value. In between, the entropy jump is finite. From the slope of the calculated phase line a maximum value of $\Delta S \sim 0.4 k_{B} \pm 0.2$ near $60 \mathrm{GPa}$ at $15 \mathrm{~K}$ can be obtained, to be compared with $0.5 k_{B}$ of the experimental slope at same pressure and temperature (see Fig. 20) and the contribution to the entropy, predicted by mean field theory, in the strong interaction limit [81], equal to $k_{B} \ln 2=0.693 k_{B}$, confirming that the entropy is an additional factor that stabilizes the ordered phase.

\subsection{Reentrant phase transitions in ortho-para mixtures}

As was shown in the preceding section, depending on the parity of the rotational quantum number $J$, solid hydrogens exhibit either pressure-driven BSP quantum phase transitions (even- $J$ species $p-\mathrm{H}_{2}$ and $o-\mathrm{D}_{2}$ ) or usual orderdisorder classical phase transitions (odd- $J$ species $o-\mathrm{H}_{2}$ and $\left.p-\mathrm{D}_{2}\right)$. Solid HD was found to possess an additional anomaly: its BSP phase transition line displays a minimum, indicating that the disordered phase is reentrant. Such a strong difference in the pressure behavior of even-, odd-, and all- $J$ species raises an intriguing question of the possible phase diagrams of their mixtures.

Two theoretical papers were devoted to this problem. We will start from a mean-field theory developed by Freiman, Tretyak, Mao and Hemley [89]. To circumvent the main difficulty which one encounters considering mixtures, the systems without a translational invariance, the authors used the following trick. It was supposed that each site of the lattice is occupied by a superposition of even- $J$ and odd- $J$ rotors. Two limiting cases are considered: mixtures at thermodynamic equilibrium, where the conversion time is small or comparable with the thermalization time, and the opposite case, frozen mixtures, when the conversion time is large compared with all other relevant times. In the former case, the fractions of even and odd species in the mixture are temperature dependent and equal to thermodynamically average concentrations; for the latter, the fraction of the species are fixed. The molecular field Hamiltonian (15) was used to describe dynamics of orientational degrees of freedom.

In the case of the frozen systems the partition function of the system is a product of the partition functions of the even and odd systems:

$$
Z=Z_{\text {even }} Z_{\text {odd }} \text {. }
$$

In this case the free energy of the system is a sum of free energies of the even and odd systems, and there is a simple relation between the order parameter of the system $\eta=\left\langle Y_{20}\right\rangle$ and order parameters of even $\eta_{\text {even }}=\left\langle Y_{20}\right\rangle_{\text {even }}$ and odd $\eta_{\text {odd }}=\left\langle Y_{20}\right\rangle_{\text {odd }}$ systems:

$$
\left\langle Y_{20}\right\rangle=(1-c)\left\langle Y_{20}\right\rangle_{\text {even }}+c\left\langle Y_{20}\right\rangle_{\text {odd }} \text {. }
$$

In the case of the equilibrium systems, the partition function of the system is the sum of the partition functions of even and odd systems:

$$
Z=Z_{\text {even }}+Z_{\text {odd }} \text {. }
$$




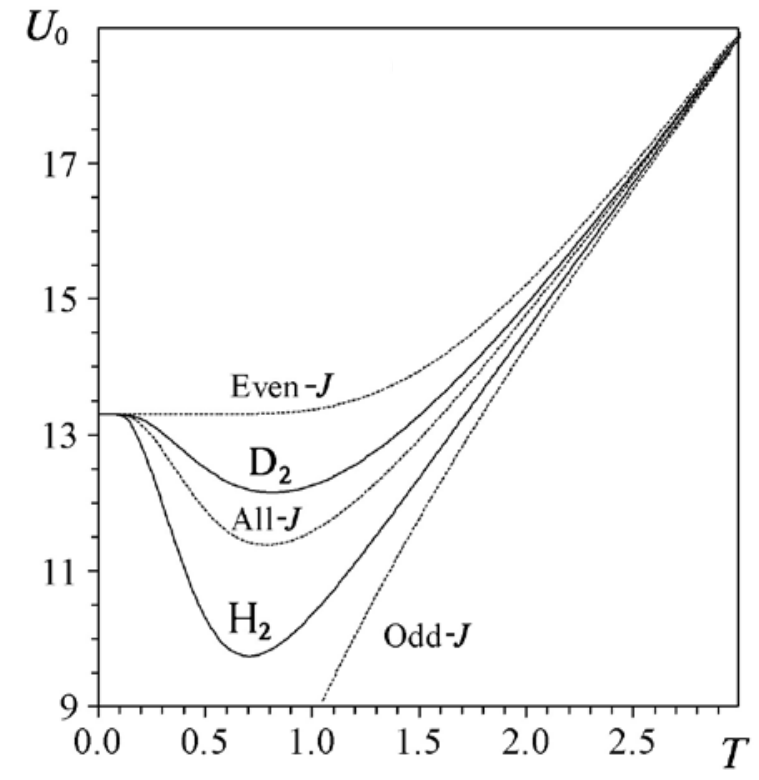

Fig. 21. Equilibrium o-p phase diagram (after Freiman et al. [89]).

In this case a simple expression relating $\eta=\left\langle Y_{20}\right\rangle$ with $\eta_{\text {even }}=\left\langle Y_{20}\right\rangle_{\text {even }}$ and odd $\eta_{\text {odd }}=\left\langle Y_{20}\right\rangle_{\text {odd }}$ does not exist.

The resulting phase diagrams are shown in Figs. 21 and 22 for the thermodynamically equilibrium and frozen even- $J$ odd- $J$ mixtures, respectively. In Fig. 21, phase diagrams for the pure even- $J$ and pure odd- $J$ systems, as well as that for the all- $J$ system, are also shown for comparison.

As one can see, there is a striking difference between these diagrams. The equilibrium even- $J$ odd- $J$ mixtures exhibit the same type of reentrant behavior as known for the all- $J$ systems (see Subsecs. 4.2, 4.3). The strongest reentrance is displayed by the even- $J$ odd- $J$ linear rotor

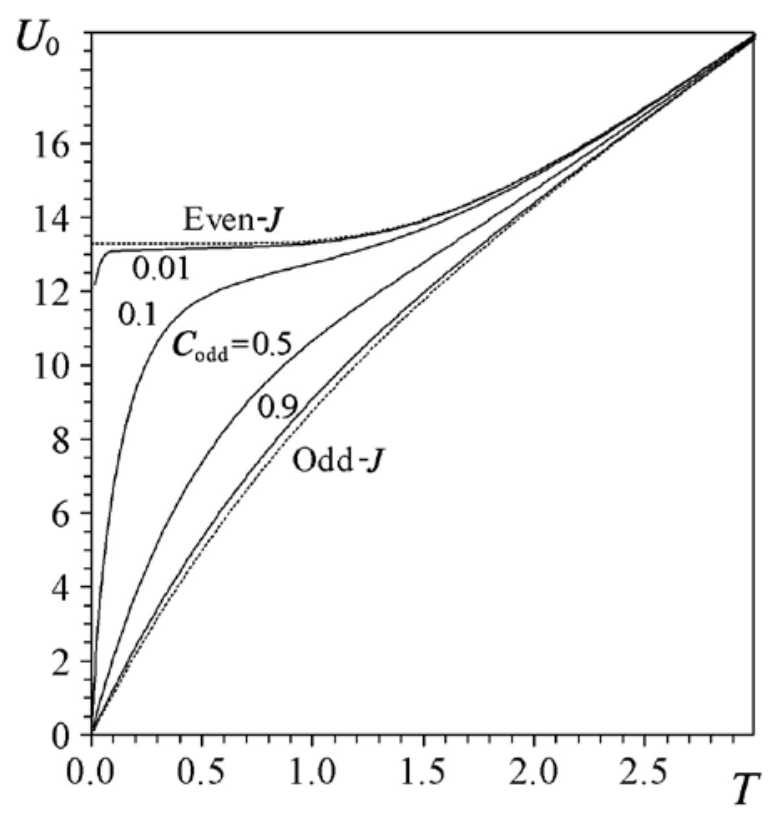

Fig. 22. Frozen $o-p$ phase diagram (after Freiman et al. [89]).

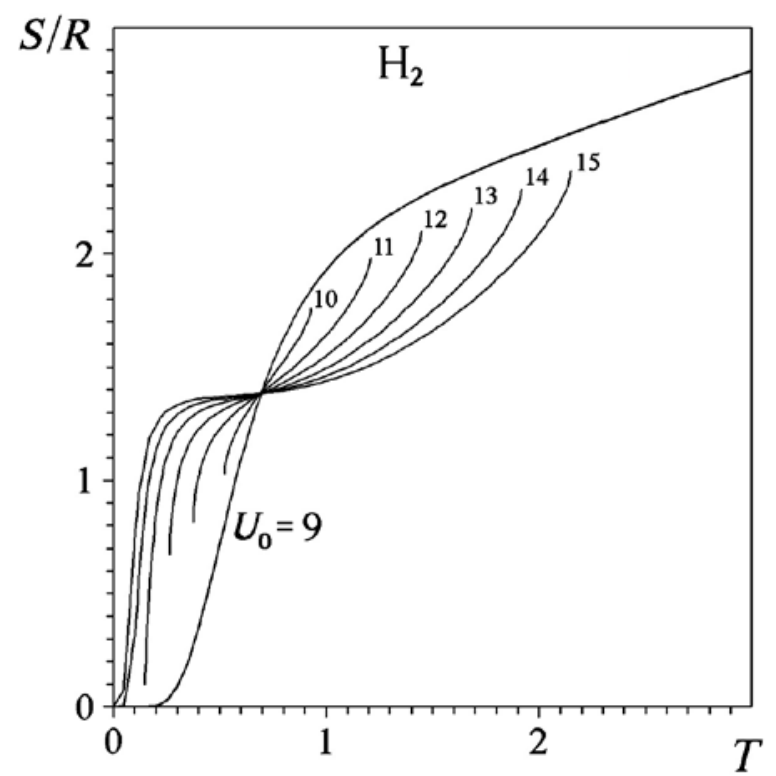

Fig. 23. Entropy of the equilibrium $o-p$ mixture of solid hydrogens (after Freiman et al. [89]).

mixture (equilibrium $o-p-\mathrm{H}_{2}$ mixture), the least one by the equilibrium o- $p$ mixture of $\mathrm{D}_{2}$ with $\mathrm{HD}$ intermediate between them. The frozen systems display qualitatively different behavior of their phase transition lines (Fig. 21). For any even- $J$ odd- $J$ compositions excluding the case of the pure even- $J$ system the phase transition lines go monotonically to the point $P=0$ with decreasing temperature. This means that the passage to the limit of the zero fraction of the odd modification is discontinuous.

An analysis of the entropy contribution to the free energy (Figs. 23, 24) furnishes an understanding of the nature of the reentrant behavior and of the distinctions between

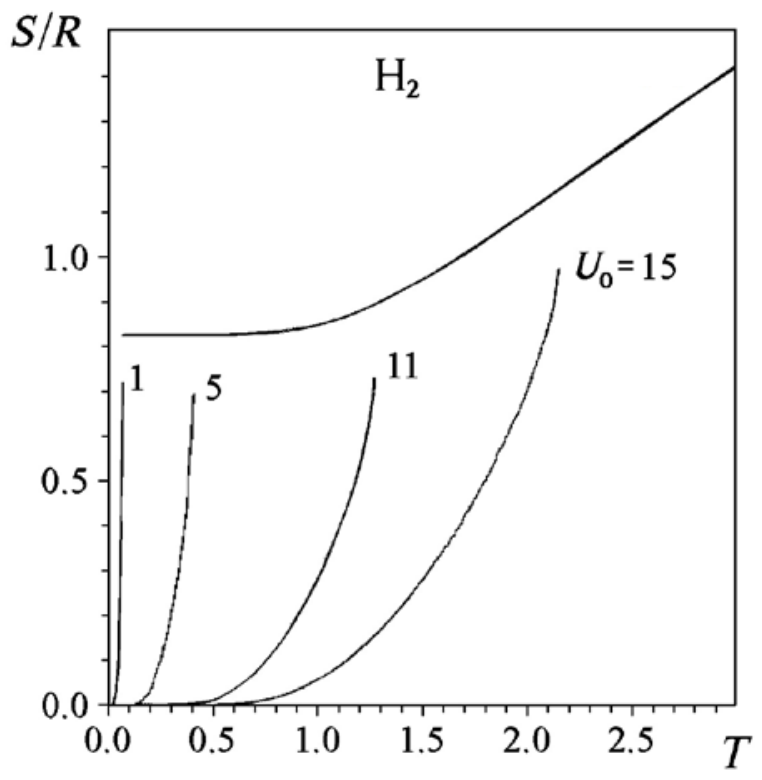

Fig. 24. Entropy of the frozen $o-p$ mixture of solid hydrogens (after Freiman et al. [89]). 


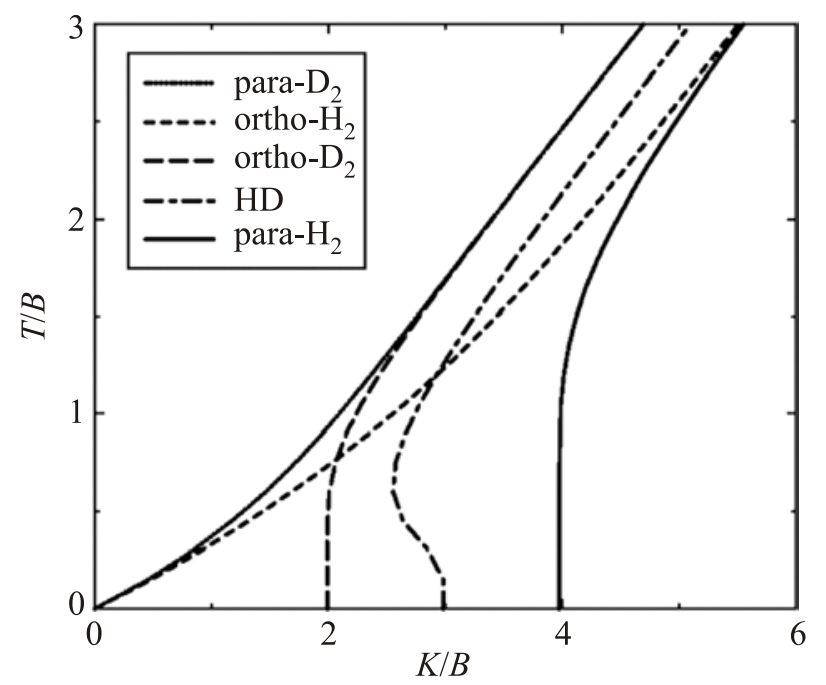

Fig. 25. Phase diagrams of the pure systems $\mathrm{H}_{2}$ and $\mathrm{D}_{2}$ at thermal equilibrium distribution calculated using multiorder parameter mean-field theory and the standard mean-field theory (MF). After Ref. 97.

the equilibrium and frozen ortho-para mixtures. As can be seen from Fig. 23, the entropy of the equilibrium and all- $J$ systems is anomalous: in the low-temperature region the entropy of the ordered phase is higher than that of the disordered phase. Thus, the entropy contribution is an additional ordering factor; its value increases with temperature and, as a result, the ordering pressure goes down as temperature increases. At a certain temperature, the entropy of the disordered phase becomes higher than that of the ordered phase and the entropy contribution turns into a disordering factor and the ordering pressure turns upward after passing the minimum point. In contrast with that, for the frozen systems no such anomaly exists for the frozen systems (see Fig. 25).

In its turn, the entropy anomaly can be traced at the microscopic level to characteristic features of the spectrum of the ordered phase (Fig. 16). For the all- $J$ system (HD) the ground state is formed by two closely spaced levels (a doublet in the strong interaction limit) as opposite to the singlet ground state of the disordered phase. As a result, there is an additional contribution $R \ln 2$ to the ordered phase entropy. At the same time, both even- and odd- $J$ pure systems have singlet ground states and their transition lines do not exhibit reentrance. The same entropy-based considerations can be applied to the case of the BSP transitions in the even- $J$ odd- $J$ mixtures of quantum linear rotors. In the equilibrium case the partition function of the homonuclear molecules is the sum of the partition functions of the even- $J$ and odd- $J$ states taken with their nuclear spin- symmetry weights. As a result, for equilibrium mixtures the ground state phase of the ordered phase is a quadruplet for $\mathrm{H}_{2}$ (in a large $V$ limit) and a octuplet for $\mathrm{D}_{2}$. Taking into account that the disordered phase entropies (at zero temperature) are zero for $\mathrm{H}_{2}$ and $R \ln 4$ for $\mathrm{D}_{2}$, additional contributions to the ordered phase entropies for $\mathrm{H}_{2}$ and $\mathrm{D}_{2}$ are $R \ln 4$ and $R \ln (4 / 3)$, respectively. This explains why the reentrance decreases in the sequence $\mathrm{H}_{2}$, $\mathrm{HD}, \mathrm{D}_{2}$. In the case of the frozen mixtures, the partition function of the mixture is a product of the partition functions of the components, each having a singlet ground state, with no reentrance resulting.

Hetényi et al. [97], using a multi-parametrized meanfield (MF) formalism, also found that the equilibrium even- $J$ odd- $J$ mixtures have reentrant phase diagrams. The main advantage of their formalism is that contrary to the previous work where it was supposed that each site of the lattice is occupied by a superposition of even- $J$ and odd- $J$ rotors the new formalism allows for onsite distinction of different spin-nuclear species that can be distributed randomly inside the lattice. This is a more realistic approximation that allows also for the inclusion of local order parameter correlation functions and therefore it is possible to differentiate short-range order from long-range order a feature that is absent in the standard MF theory where only phases of complete order or disorder are possible.

The multiorder parameter (MOP) mean-field theory is based on the trial Hamiltonian

$$
\begin{aligned}
H_{0}=B_{\text {rot }} \sum_{i=1}^{N} \hat{L}_{i}^{2}+K \sum_{i<j}^{N}\left(\frac{R_{0}}{R_{i j}}\right)^{5}\left[Y_{20}\left(\Omega_{i}\right)-\frac{1}{2} \gamma_{i}\right] \times \\
\quad \times C(224 ; 00) \gamma_{j} Y_{40}^{*}\left(\Omega_{i j}\right),
\end{aligned}
$$

where $K$ is the coupling strength, $C(224 ; 00)$ is a ClebschGordan coefficient, $\Omega_{i j}$ denote the direction of the vector connecting rotors $i$ and $j$ and $\gamma_{i}$ are parameters. Variation of the free energy leads to the self-consistent expression

$$
\gamma_{i}=\left\langle Y_{20}\left(\Omega_{i}\right)\right\rangle_{0}
$$

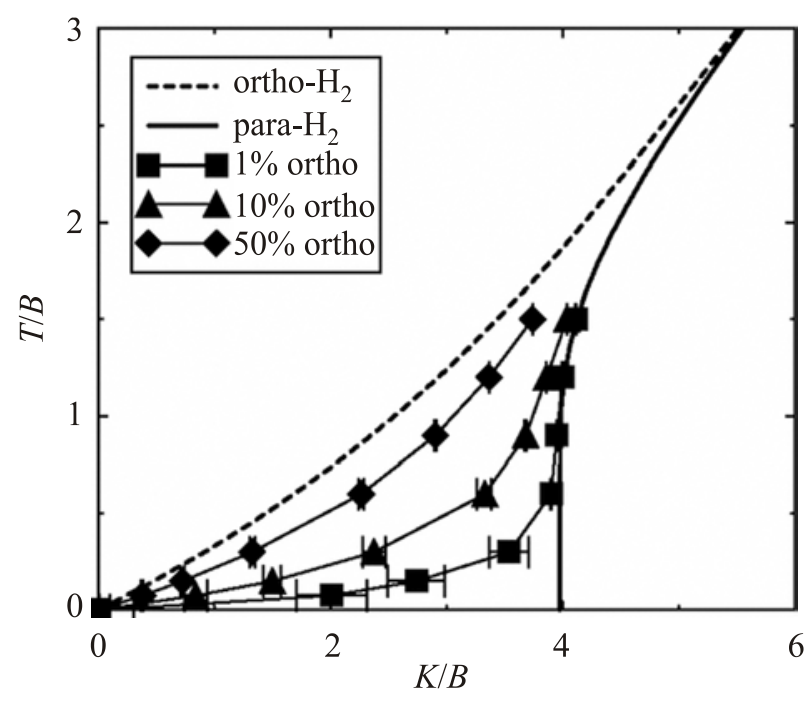

Fig. 26. Multiorder parameter MF phase diagrams for coupled quadrupolar rotor models corresponding to solid molecular hydrogen frozen at various ortho concentrations. After Ref. 97. 
Since the trial Hamiltonian in Eq. (24) is a sum of singlerotor Hamiltonians, it follows that for each $i$ in Eq. (25) the average needs to be performed over the corresponding coordinate $i$ only. Ortho-para distinction can be implemented by restricting a particular average to be over odd- $J$ or even- $J$ states. This formalism was applied to a system of coupled quadrupolar rotors whose centers of mass form a fcc lattice. The methodology for the self-consistent calculation of the parameters $\left\{\gamma_{i}\right\}$ is described in Ref. 97. Order is then signaled by nonzero solutions for $\gamma_{i}$, disorder by solutions in which all $\gamma_{i}$ are identically zero.

The orientational ordering of the system with ortho-para concentrations at thermal equilibrium is shown in Fig. 26 along with the phase diagrams of the pure systems. For $\mathrm{H}_{2}$ and $\mathrm{D}_{2}$ the calculated phase diagrams via the MOP meanfield theory and via the standard MF theory are very similar (see Fig. 21) and the reentrance phase line for $\mathrm{H}_{2}$ and $\mathrm{D}_{2}$ is also obtained within this approximation. In Fig. 26 the results of the formalism presented above are shown for solid $\mathrm{H}_{2}$ at a frozen concentration of ortho species. As in the case of standard MF as the ortho concentration is decreased the system tends towards disorder entering the ordered state at higher coupling constants for a given temperature (see also Fig. 22). Nevertheless a noteworthy result is obtained with this new formalism. As one can see from Fig. 26 even at an ortho concentration of $1 \%$ the system enters an ordered state at coupling constants quite different from that of pure parahydrogen, and that for any ortho concentration the ground state is always ordered. This result is not observed in the case of standard MF theory (see the 0.01 curve in Fig. 22). The reason for this difference is because the orientational order is short ranged at low temperatures. This feature can be just capture with the MOP mean-field approximation where it is possible to differentiate short-range order from long-range order while only long-range order could be observed in standard MF

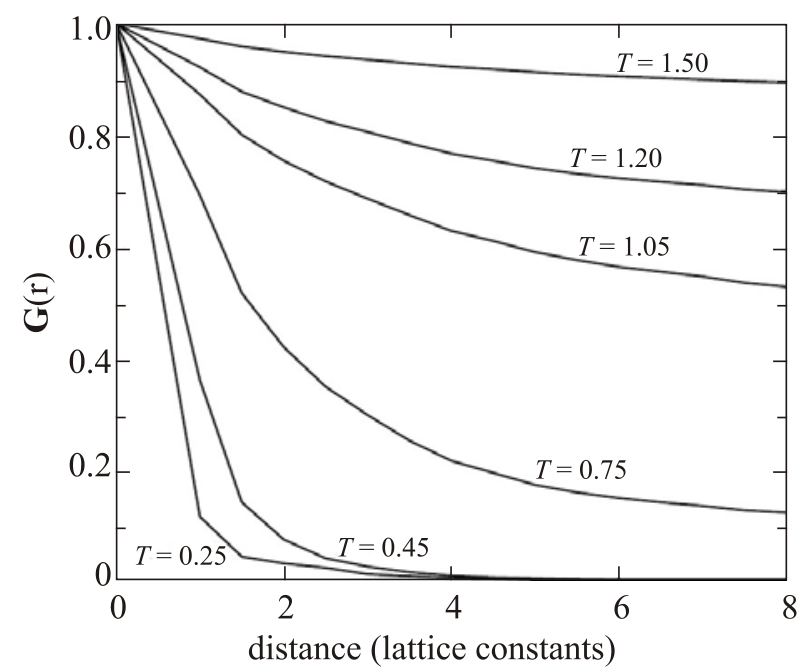

Fig. 27. Correlation functions along the phase boundary of the solid $\mathrm{H}_{2}$ at thermal equilibrium. After Ref. 97. theory. In order to assess the nature of the ordering the correlation functions $G(r)$ of the local order parameters $\left\{\gamma_{i}\right\}$ was calculated using the expression.

$$
G(r)=\left\langle[\gamma(0) \gamma(r)]^{2}\right\rangle \text {. }
$$

The correlation functions for different temperatures along the reentrant phase diagram (Fig. 25) are shown for $\mathrm{H}_{2}$ in Fig. 27. As the temperature increases correlation increases along the phase boundary. At high temperatures $(T / B>1)$ the order is definitely long range. The short-range order is expected to be present at low- $T$ up to $T / B<0.75$. The increases of the correlation along the phase boundary with increasing temperature indicates the onset of longrange order. The onset of long-range order is due to the fact that ortho-para distinction ceases as temperature and coupling constant (pressure) are increased.

\section{Conclusion}

In this Section we summarize the results discussed in this review article with a stress on open experimental and theoretical problems that still remain to be solved. These problems span the whole region of the phase diagram. Naturally, the most "hot" are problems at high-pressure frontiers.

In the Introduction (Sec. 1) we have discussed the constraints which quantum mechanics impose on the possible link between rotational states of the hydrogen molecules and their total nuclear spin. This symmetry-related link gives rise the existence of ortho- and paramodifications displaying large differences in the solid-state properties.

In Sec. 2 we reviewed experimental and theoretical works devoted to studies of the ortho-para conversion in solid hydrogen and deuterium with the emphasis on the high-pressure studies. The highest pressures for which the experimental conversion rate data exist are at present $58 \mathrm{GPa}$. The extension of these studies to higher pressures would provide a critical test of the conversion mechanisms proposed by theory. It will need further theoretical studies to validate the conception of ortho-para states at ultra-high pressures.

In Sec. 3 we discuss two of numerous effects displaying by the $J=0-J=1$ mixtures of solid hydrogen and deuterium: the effect of $J=1$ admixtures on the molar volume, and the hcp lattice distortion.

Hydrogen is a quantum solid and is therefore highly compressible. Due to the large compressibility, the weak quadrupole-quadrupole forces related with $J=1$ species have a non-negligible effect on the molar volume.

As compared with fcc, the hcp lattice has an additional degree of freedom associated with the $c / a$ ratio. In this Section we discuss the deviation of the axial ratio for the $J=0-J=1$ mixtures from the ideal value $\sqrt{8 / 3} \approx 1.633$. It was shown that the presence of $J=1$ molecules increases the hcp lattice distortion by two order of magnitude. 
Section 4 is devoted to the peculiarities of the phase diagram of solid HD. Near $50 \mathrm{GPa}$ it exhibits a reentrant phase transition where a rotationally ordered («broken symmetry») crystalline phase surprisingly transforms into a orientationally disordered high-symmetry phase upon cooling. The molecular-field theory gives the qualitative reason for reentrance: the higher entropy of the broken symmetry phase, due to the inequivalence of $\mathrm{H}$ and $\mathrm{D}$, as opposed to the low entropy of the high-symmetry phase where the rotational melting is quantum mechanical - a Pomeranchuk-like mechanism. The entropy jump across the transition is found to be comparable with $\ln 2$, the value expected for the Pomeranchuk mechanism. Path Integral Monte Carlo calculations give a reentrant phase boundary in good agreement with experiment.

On the ground of the similar entropy-based considerations the molecular-field theory forecasts that the brokenphase transitions in thermodynamically equilibrium orthopara mixtures of homonuclear species of $\mathrm{H}_{2}$ and $\mathrm{D}_{2}$ should exhibit reentrant phase transition lines. This prediction makes the respective experimental studies and the development of theory of the BSP transition in such systems beyond the MF approximation important actual problem

In Sec. 5 we listed a number of problems both in experiment and in theory that still remain to be solved. These problems span the whole region of the phase diagram. Naturally, the most "hot" are problems at the high-pressure frontiers.

The authors are deeply indebted to Erio Tosatti and Mikhail Strzhemechny for numerous fruitful discussions.

1. I.F. Silvera, Rev. Mod. Phys. 52, 393 (1980).

2. J. Van Kranendonk, Solid Hydrogen, Plenum, New York (1983).

3. Physics of Cryocrystals, V.G. Manzhelii and Yu.A. Freiman (eds.), AIP Press, New York (1997).

4. E. Cremer and M. Polanyi, Trans. Faraday Soc. 28, 435 (1932); Z. Phys. Chem. B 21, 459 (1933).

5. L. Farkas, Ergeb. Exacten Naturwiss. 12, 163 (1933).

6. A. Driessen, E. van der Poll, and I.F. Silvera, Phys. Rev. B 30, 2517 (1984).

7. G. Ahlers, J. Chem. Phys. 40, 3123 (1964).

8. P. Pedroni, H. Meyer, F. Weinhaus, and D. Haase, Solid State Commun. 14, 279 (1974).

9. F. Schmidt, Phys. Rev. B 10, 4480 (1974).

10. R.F. Buzerak, Ph.D. Thesis, Duke University (1975).

11. R. Jochemsen, Ph.D. thesis, Univ. of Amsterdam (1978).

12. I.F. Silvera, P.J. Berkhout, and L.M. van Aernsbergen, J. Low Temp. Phys. 35, 611 (1979).

13. R.J. Hemley, A.F. Goncharov, H.-k. Mao, J.H. Eggert, and E. Karmon, J. Low Temp. Phys. 110, 75 (1998).

14. J.H. Eggert, E. Karmon, R.J. Hemley, H.-k. Mao, and A.F. Goncharov, Proc. Natl. Acad. Sci. USA 96, 269 (1999).

15. M. Pravica, and I.F. Silvera, Phys. Rev. Lett. 81, 4180 (1998).

16. M.A. Strzhemechny and R.J. Hemley, Phys. Rev. Lett. 85, 5595 (2000).
17. K. Motizuki and T. Nagamiya, J. Phys. Soc. Jpn. 11, 93 (1956).

18. A.J. Berlinsky and W.N. Hardy, Phys. Rev. B 8, 5013 (1973).

19. A.J. Berlinsky, Phys. Rev. B 12, 1482 (1975).

20. M.A. Strzhemechny, R.J. Hemley, H.-k. Mao, A.F. Goncharov, and J.H. Eggert, Phys. Rev. B 66, 014103 (2002).

21. K. Motizuki, J. Phys. Soc. Jpn. 12, 163 (1957).

22. W.N. Hardy and A.J. Berlinsky, Phys. Rev. B 8, 4996 (1973).

23. Yu.Ya. Milenko and R.M. Sibileva, Fiz. Nizk. Temp. 1, 796 (1975) [Sov. J. Low Temp. Phys. 1, 382 (1975)].

24. P.J. Berkhout, J. Th. Minneboo, and I.F. Silvera J. Low Temp. Phys. 32, 401 (1978).

25. M. Calkins, R. Banke, X. Li, and H. Meyer, J. Low Temp. Phys. 65, 47 (1986).

26. M.I. Bagatskii, A.I. Krivchikov, V.G. Manzhelii, I.Ya. Minchina, and P.I. Muromtsev, Fiz. Nizk. Temp. 13, 1001 (1987) [Sov. J. Low Temp. Phys. 13, 571 (1987)].

27. M.A. Strzhemechny and O.I. Tokar, Fiz. Nizk. Temp. 11, 813 (1985) [Sov. J. Low Temp. Phys. 11, 446 (1985)].

28. M.A. Strzhemechny and R.J. Hemley, Fiz. Nizk. Temp. 29, 941 (2003) [Low Temp. Phys. 29, 703 (2003)].

29. I.F. Silvera, J. Low Temp. Phys. 112, 237 (1998).

30. I.F. Silvera and M. Pravica, J. Phys.: Condens. Matter 10, 11169 (1998).

31. Y. Akahama, M. Nishimura, H. Kawamura, N. Hirao, Y. Ohishi, and K. Takemura, Phys. Rev. B 82, 060101(R) (2010).

32. N.W. Ashcroft, J. Non-Cryst. Solids 156, 621 (1993).

33. I.F. Silvera, W.N. Hardy, and J.P. McTague, Phys. Rev. B 8, 1578 (1972).

34. A. Driessen, J.A. de Waal, and I.F. Silvera, J. Low Temp. Phys. 34, 255 (1979).

35. R. Howard, Phys. Lett. A 32, 37 (1970).

36. P. Schwerdtfeger, N. Gaston, R.P. Krawczyk, R. Tonner, and G.E. Moyano, Phys. Rev. B 73, 064112 (2006).

37. F.H. Stillinger, J. Chem. Phys. 115, 5208 (2001).

38. Yu.A. Freiman, S.M. Tretyak, A. Grechnev, A.F. Goncharov, J.S. Tse, D. Errandonea, H.-k. Mao, and R.J. Hemley, Phys. Rev. B 80, 094112 (2009).

39. Yu.A. Freiman, S.M. Tretyak, F. Jezowski, and R.J. Hemley, J. Low Temp. Phys. 126, 703 (2002).

40. Yu.A. Freiman, Alexei Grechnev, S.M. Tretyak, Alexander Goncharov, and Russell J. Hemley, Phys. Rev. B 86, 014111 (2012).

41. A. Grechnev, S.M. Tretyak, and Yu.A. Freiman, Fiz. Nizk. Temp. 36, 423 (2010) [Low Temp. Phys. 36, 333 (2010)].

42. W.H. Keesom, J. de Smedt, and H.H. Moon, Leiden Commun. 19, Suppl. No. 69, No. 209d (1929/1930).

43. I.N. Krupskii, A.I. Prokhvatilov, and G.N. Shcherbakov, Fiz. Nizk. Temp. 9, 83 (1983) [Sov. J. Low Temp. Phys. 9, 42 (1983)]; ibid. 10, 5 (1984) [ibid. 10, 1 (1984)].

44. S.N. Ishmaev, I.P. Sadikov, A.A. Chernyshov, B.A. Vindryaevskii, V.A. Sukhoparov, A. Telepnev, and G.V. Kobelev, Sov. Phys. JETP 57, 228 (1983).

45. S.N. Ishmaev, I.P. Sadikov, A.A. Chernyshov, B.A. Vindryaevskii, V.A. Sukhoparov, A. Telepnev, G.V. Kobelev, and R.A. Sadikov, Sov. Phys. JETP 62, 721 (1985). 
46. A.F. Goncharov, M.A. Strzhemechny, H.-k. Mao, and R.J. Hemley, Phys. Rev. B 63, 064304 (2001).

47. Yu.A. Freiman, S.M. Tretyak, Alexei Grechnev, Alexander F. Goncharov, and Russell J. Hemley, Phys. Rev. B 90, 024501 (2014).

48. P. Loubeyre, R. LeToullec, D. Hausermann, M. Hanfland, R.J. Hemley, H.-k. Mao, and L.W. Finger, Nature 383, 702 (1996).

49. R.J. Hemley, H.-k. Mao, L.W. Finger, A.P. Jephcoat, R.M. Hazen, and C.S. Zha, Phys. Rev. B 42, 6458 (1990).

50. H.-k. Mao and Russell J. Hemley, Rev. Mod. Phys. 66, 671 (1994).

51. H. Kawamura, Y. Akahama, S. Umemoto, K. Takemura, Y. Ohishi, and O. Shimomura, J. Phys.: Condens. Matter 14, 10407 (2002); ibid. 86, 014111 (2012).

52. Yu.A. Freiman, S.M. Tretyak, Alexander F. Goncharov, H.-k. Mao, and Russell J. Hemley, Fiz. Nizk. Temp. 37, 1302 (2011) [Low Temp. Phys. 37, 1038 (2011)].

53. K. Mendelssohn, M. Ruhemann, and F. Simon, Z. Phys Chem. B 15, 121 (1931).

54. K. Schaefer, Z. Phys. Chem. B 42, 380 (1939); ibid. B 45, 451 (1940).

55. T. Nagamiya and H. Kisi, Busseiron Kenkyu (in Japanese) 39, 64 (1951).

56. T. Nakamura, Prog. Theor. Phys. (Kyoto) 14, 135 (1955).

57. R.W. Hill and B.W. Richetson, Philos. Mag. 45, 277 (1954).

58. R.J. Roberts, E. Rojas, and J.G. Daunt, J. Low Temp. Phys. 24, 265 (1976).

59. W.H. Orttung, J. Chem. Phys. 36, 652 (1962).

60. J. van Kranendonk and V.F. Sears, Can. J. Phys. 44, 313 (1966).

61. A.B. Harris and E.R. Hunt, Phys. Rev. Lett. 16, 845 (1966).

62. W.N. Hardy and J.R. Gaines, Phys. Rev. Lett. 19, 1417 (1967).

63. J.H. Constable and J.R. Gaines, Solid State Commun. 9, 155 (1977).

64. R. Schweizer, S. Washburn, and H. Meyer, J. Low Temp. Phys. 37, 289 (1979).

65. W.N. Hardy, A.J. Berlinsky, and A.B. Harris, Can. J. Phys. 55, 1150 (1977).

66. J.C. Raich and L.B. Kanney, J. Low Temp. Phys. 28, 95 (1977).

67. S. Luryi and J. van Kranendonk, Can. J. Phys. 57, 933 (1979).

68. R.M. Dickinson, T.J. Byers, and T. Oka, J. Low Temp. Phys. 102, 241 (1996).

69. A.I. Prokhvatilov, M.A. Strzhemechny, and G.N. Shcherbakov, Fiz. Nizk. Temp. 19, 622 (1993) [Low Temp. Phys. 19, 445 (1993)].

70. I.N. Krupskii, A.I. Prokhvatilov, and G.N. Shcherbakov, Fiz. Nizk. Temp. 9, 858 (1983) [Sov. J. Low Temp. Phys. 9, 446 (1983)].
71. M.A. Strzhemechny, Phys. Rev. B 73, 174301 (2006).

72. J. Bigeleisen, J. Chem. Phys. 39, 769 (1963).

73. M. Trefler and H.P. Gush, Phys. Rev. Lett. 20, 703 (1968).

74. M.A. Strzhemechny and R.J. Hemley, Phys. Rev. B 91, 144102 (2015).

75. Yu.A. Freiman and M.A. Strzhemechny, In: Physics of Cryocrystals [3], Ch. 17, Kinetic Properties, AIP Press, NY (1997), p. 489.

76. A. Chijioke and I.F. Silvera, Phys. Rev. Lett. 97, 255701 (2006).

77. L. Cui, N.H. Chen, and I.F. Silvera, Phys. Rev. B 51, 14987 (1995).

78. F. Moshary, N.H. Chen, and I.F. Silvera, Phys. Rev. Lett. 71, 3814 (1993).

79. H.E. Lorenzana, I.F. Silvera, and K.A. Goettel, Phys. Rev. Lett. 64, 1939 (1990).

80. Yu.A. Freiman, V.V. Sumarokov, A.P. Brodyanskii, and A. Jezowski, J. Phys.: Condens. Matter 3, 3855 (1991).

81. A.P. Brodyanskii, V.V. Sumarokov, Yu.A. Freiman, and A. Jezowski, Fiz. Nizk. Temp. 19, 520 (1993) [Low Temp. Phys. 19, 368 (1993)].

82. Yu.A. Freiman, and S.M. Tretyak, A. Jezowski, and R.J. Hemley, J. Low Temp. Phys. 113, 723 (1998).

83. Yu.A. Freiman, S.M. Tretyak, and Andrzej Jezowski, J. Low Temp. Phys. 110, 147 (1998).

84. Hyeondeok Shin and Yongkyung Kwon, J. Korean Phys. Soc. 54, 1582 (2009).

85. Yanier. Crespo, Alessandro Laio, Giuseppe E. Santoro, and Erio Tosatti, Phys. Rev. B 84, 144119 (2011).

86. R.J. Hemley and H.-k. Mao, Phys. Rev. Lett. 61, 857 (1988).

87. R.J. Hemley and H.-k. Mao, Phys. Rev. Lett. 63, 1393 (1989).

88. L. Cui, N.H. Chen, S.J. Jeon, and I.F. Silvera, Phys. Rev. Lett. 72, 3048 (1994).

89. Yu.A. Freiman, S.M. Tretyak, H.-k. Mao, and R.J. Hemley, J. Low Temp. Phys. 139, 765 (2005).

90. V.V. Sumarokov and Yu.A. Freiman, Fiz. Nizk. Temp. 13, 197 (1987) [Sov. J. Low Temp. Phys. 13, 109 (1987)].

91. D.M. Ceperley, Rev. Mod. Phys. 67, 279 (1995).

92. T. Cui, E. Cheng, B.J. Alder, and K.B. Whaley, Phys. Rev. B 55, 12253 (1997).

93. J. Schaefer and E. Köhler, Z. Phys. B 13, 217 (1989).

94. P.G. Burton and U.E. Senff, J. Chem. Phys. 76, 6073 (1982).

95. K.J. Runge, M.P. Surh, C. Mailhiot, and E.L. Pollock, Phys. Rev. Lett. 69, 3527 (1992).

96. M.P. Surh, K.J. Runge, T.W. Barbee, E.L. Pollock, and C. Mailhiot, Phys. Rev. B 55, 11330 (1997).

97. B. Hetényi, S. Scandolo, and E. Tosatti, Phys. Rev. Lett. 94, 125503 (2005). 